\title{
Two-Weight Orlicz Type Integral Inequalities for the Maximal Operator ${ }^{1}$
}

\author{
C.J. Neugebauer*
}

Department of Mathematics, Purdue University, West Lafayette, in 47907-1395, USA

\begin{abstract}
We present a two-weight Orlicz-type integral inequality for the maximal operator which characterizes $(u, v) \in A_{p}$.
\end{abstract}

Keywords: Maximal operator, two-weights.

\section{INTRODUCTION}

In this paper we will study integral inequalities of the type

$\int_{R^{n}} \Phi\left(\operatorname{Mf}(x)^{p}\right) u(x) d x \leq c_{1} \int_{R^{n}} \Psi\left(c_{2}|f(x)|^{p}\right) v(x) d x$,

where $\operatorname{Mf}(\mathrm{x})=\sup _{\mathrm{x} \in \mathrm{Q}} \frac{1}{|\mathrm{Q}|} \int_{\mathrm{Q}}|\mathrm{f}(\mathrm{t})| \mathrm{dt}$ is the Hardy-Littlewood maximal operator, and we ask for condtions on $\Phi, \Psi: R_{+} \rightarrow R_{+}$such that (1) holds if and only if $(u, v) \in A_{p}$.

We say that $(\mathrm{u}, \mathrm{v}) \in \mathrm{A}_{\mathrm{p}}$ if $\frac{1}{|\mathrm{Q}|} \int_{\mathrm{Q}} \mathrm{u}\left(\frac{1}{|\mathrm{Q}|} \int_{\mathrm{Q}} \mathrm{v}^{1-\mathrm{p}^{\prime}}\right)^{\mathrm{p}-1} \leq \mathrm{c}<\infty, 1<\mathrm{p}<\infty$, and $\mathrm{Mu}(\mathrm{x}) \leq \mathrm{cv}(\mathrm{x})$, if $\mathrm{p}=1$. These weight classes were introduced by Muckenhoupt [4] and Muckenhoupt and Wheeden [5] to study (1) when $\Phi(t)=\Psi(t)=t$. If $1<\mathrm{p}<\infty$ and $\mathrm{u}=\mathrm{v} \in \mathrm{A}_{\mathrm{p}}$, (1) holds for $\Phi(\mathrm{t})=\Psi(\mathrm{t})=\mathrm{t}$, but not if $\mathrm{p}=1$. Also for each $1 \leq \mathrm{p}<\infty$ there exists a pair $(\mathrm{u}, \mathrm{v}) \in \mathrm{A}_{\mathrm{p}}$ so that (1) fails in the special case $\Phi(t)=\Psi(t)=t \quad[3$, p. 395]. In these exceptional cases we have a weak type inequality. An excellent reference is the book by J.Garcia-Cuerva and J.L.Rubio de Francia [3]. We refer the reader interested in the current state of the two-weight theory to the recent book [1] by Cruz-Uribe, Martell, and Pérez.

The restrictions on $\Phi, \Psi$ are: $\Phi(\mathrm{t})=\int_{0}^{\mathrm{t}} \mathrm{a}(\mathrm{s}) \mathrm{ds}, \Psi(\mathrm{t})=\int_{0}^{\mathrm{t}} \mathrm{b}(\mathrm{s}) \mathrm{ds}$ with $a, b: R_{+} \rightarrow R_{+}$satisfying

$\int_{0}^{\mathrm{s}} \frac{\mathrm{a}(\mathrm{t})}{\mathrm{t}} \mathrm{dt} \leq \mathrm{c}^{\prime} \mathrm{b}\left(\mathrm{c}^{\prime \prime} \mathrm{s}\right), 0<\mathrm{s}<\infty$.

Note that this excludes the classical case $\Phi(t)=\Psi(t)=t$. If (*) holds, we say that $\Phi, \Psi$ are $\left(\mathrm{c}^{\prime}, \mathrm{c}^{\prime \prime}\right)$-related.

*Address correspondence to this author at the Department of Mathematics, Purdue University, West Lafayette, in 47907-1395, USA;

E-mail: neug@math.purdue.edu

${ }^{1} 1991$ Mathematics Subject Classification, 42B25, 42B35.
We are now ready to state our main result whose proof will be given in section 3 .

Theorem 1 The following statements are equivalent for $1 \leq \mathrm{p}<\infty$.

(2) For each $\Phi$ and $\Psi$ which are $\left(c^{\prime}, c^{\prime \prime}\right)$-related, we have $\int_{R^{n}} \Phi\left(\right.$ Mf $\left.^{\mathrm{p}}\right) \mathrm{u} \leq \mathrm{c}_{1} \int_{\mathrm{R}^{\mathrm{n}}} \Psi\left(\mathrm{c}_{2}|\mathrm{f}|^{\mathrm{p}}\right) \mathrm{v}$,

for all $\mathrm{f}: \mathrm{R}^{\mathrm{n}} \rightarrow \mathrm{R}$, where the constamts $\mathrm{c}_{1}, \mathrm{c}_{2}$ depend only on $c^{\prime}, c^{\prime \prime}$ and $p$.

(3) We have $(u, v) \in A_{p}$.

Remark: In the Lebesgue measure case - $\mathrm{u}=\mathrm{v}=1$ integral inequalities related to (2) can be found in [6]. It should be noted that $\mathrm{p}=1$ is not excluded.

In section 4 we will examine in what sense the condition $\int_{0}^{t} \frac{a(s)}{s} d s \leq c^{\prime} b\left(c^{\prime \prime} t\right)$ is also necessary for Theorem 1, and in section 5 we will examine the extrapolation problem: when is it possible to replace p by $p-\varepsilon$ in (2). In sections 6 and 7 we will study the iterated maximal operator and its relation to extrapolation. In section 8 we will collect some unusual and surprising integral inequalities for $\mathrm{Mf}$ obtained by choosing $\Phi, \Psi$ and applying Theoren 1 .

A final comment is in order. I have dedicated this paper to the memory of Richard A. Hunt who made significant contributions to the theory of $A_{p}$-weights and to whom I am indebted for introducing me to this subject some 40 years ago.

\section{A TWO-WEIGHT DISTRIBUTIONAL INEQUALITY}

For convenience all our functions will be non-negative: $\mathrm{f}: \mathrm{R}^{\mathrm{n}} \rightarrow \mathrm{R}_{+}$.

The distributional inequality below for $\mathrm{u}=\mathrm{v}=1$ - the Lebesgue measure case - and a sublinear operator $\mathrm{T}$ instead of $\mathrm{M}$ is equivalent with saying that $\mathrm{T}$ is both weak-type (p,p) and of type $(\infty, \infty)[11, \mathrm{p} .103]$. 
Theorem 2 The following statements are equivalent for $1 \leq \mathrm{p}<\infty$.

(4) There exists $0<c_{0}<\infty$ such that for every f: $R_{n} \rightarrow R_{+}$ we have for $0<\mathrm{t}<\infty$

$\mathrm{u}\{\mathrm{x}: \operatorname{Mf}(\mathrm{x})>\mathrm{t}\} \leq \frac{\mathrm{c}_{0}}{\mathrm{t}^{\mathrm{p}}} \int_{\mathrm{t} / \mathrm{c}_{0}}^{\infty} \mathrm{v}\{\mathrm{x}: \mathrm{f}(\mathrm{x})>\mathrm{s}\} \mathrm{s}^{\mathrm{p}-1} \mathrm{ds}$.

(5) We have $(u, v) \in A_{p}$.

Proof. Apart from a minor detail, the proof follows the standard covering argument and we include it for the benefit of the reader.

$(5) \rightarrow(4)$. We may assume that $M$ is the centered maximal operator

$\operatorname{Mf}(\mathrm{x})=\sup \frac{1}{|\mathrm{Q}|} \int_{\mathrm{Q}} \mathrm{f}(\tau) \mathrm{d} \tau$,

where the sup is extended over all cubes $\mathrm{Q}$ centered at $\mathrm{x}$. We consider the case $1<\mathrm{p}<\infty$ first. Fix $\mathrm{f}: \mathrm{R}_{\mathrm{n}} \rightarrow \mathrm{R}_{+}$, and for $0<\mathrm{t}<\infty$ let $\mathrm{f}=\mathrm{f}^{\mathrm{t}}+\mathrm{f}_{\mathrm{t}}$, where

$$
\mathrm{f}^{\mathrm{t}}(\mathrm{x})=\left\{\begin{array}{cc}
0, & f(x) \leq t / 2 \\
\mathrm{f}(\mathrm{x}), & f(x)>t / 2 .
\end{array}\right.
$$

Then $\quad \operatorname{Mf}(\mathrm{x}) \leq \mathrm{Mf}^{\mathrm{t}}(\mathrm{x})+\mathrm{Mf}_{\mathrm{t}}(\mathrm{x}) \quad$ so that $\{\mathrm{Mf}>\mathrm{t}\} \subset\left\{\mathrm{Mf}^{\mathrm{t}}>\mathrm{t} / 2\right\} \equiv \mathrm{E}_{\mathrm{t}}$. Let $\mathrm{E}_{\mathrm{tN}}=\mathrm{E}_{\mathrm{t}} \cap\{\mathrm{x}:|\mathrm{x}| \leq \mathrm{N}\}$. We can now apply the Besicovitch covering Theorem and obtain cubes $\left\{Q_{j}\right\}$ satisfying

$$
\mathrm{E}_{\mathrm{tN}} \subset \cup \mathrm{Q}_{\mathrm{j}},\left|\mathrm{Q}_{\mathrm{j}}\right| \leq \frac{2}{\mathrm{t}} \int_{\mathrm{Q}_{\mathrm{j}}} \mathrm{f}^{\mathrm{t}}, \sum \chi_{\mathrm{Q}_{\mathrm{j}}} \leq \mathrm{c}<\infty .
$$

Then

$$
\begin{aligned}
& u\left(E_{t N}\right) \leq \sum u\left(Q_{j}\right) \leq \frac{c}{t^{p}} \sum \frac{u\left(Q_{j}\right)}{\left|Q_{j}\right|^{p}}\left(\int_{Q_{j}} f^{t} v^{1 / p_{v}-1 / p}\right)^{p} \\
& \leq \frac{\mathrm{c}}{\mathrm{t}^{\mathrm{p}}} \sum \frac{\mathrm{u}\left(\mathrm{Q}_{\mathrm{j}}\right)}{\left|\mathrm{Q}_{\mathrm{j}}\right|^{\mathrm{p}}} \int_{\mathrm{Q}_{\mathrm{j}}}\left(\mathrm{f}^{\mathrm{t}}\right)^{\mathrm{p}} \mathrm{v} \cdot\left(\int_{\mathrm{Q}_{\mathrm{j}}} \mathrm{v}^{1-\mathrm{p}^{\prime}}\right)^{\mathrm{p}-1} \\
& \leq \frac{\mathrm{c}}{\mathrm{t}^{\mathrm{p}}} \int_{\{\mathrm{f} \geq \mathrm{t} / 2\}} \mathrm{f}^{\mathrm{p}} \mathrm{v} . \\
& \text { If } A_{t}=\{x: f(x) \geq t / 2\} \text {, then }
\end{aligned}
$$$$
\mathrm{u}\left(\mathrm{E}_{\mathrm{tN}}\right) \leq \frac{\mathrm{c}}{\mathrm{t}^{\mathrm{p}}} \int_{\mathrm{R}^{\mathrm{n}}}\left(\mathrm{f} \chi_{\mathrm{A}_{\mathrm{t}}}\right)^{\mathrm{p}} \mathrm{v}=\frac{\mathrm{c}}{\mathrm{t}^{\mathrm{p}}} \int_{0}^{\infty} \mathrm{v}\left\{f \chi_{\mathrm{A}_{\mathrm{t}}}>\mathrm{s}\right\} \mathrm{s}^{\mathrm{p}-1} d s
$$$$
=\frac{c}{t^{p}}\left(\int_{t / 2}^{\infty} v\{f>s\} s^{p-1} d s+v\left(A_{t}\right) \int_{0}^{t / 2} s^{p-1} d s\right) \text {. }
$$

It is clear that for some constant $\mathrm{c}$

$$
c \int_{t / 4}^{t / 2} v\{f>s\} s^{p-1} d s \geq v\left(A_{t}\right) \int_{0}^{t / 2} s^{p-1} d s,
$$

and hence for some constant $c_{0}$
$\mathrm{u}\left(\mathrm{E}_{\mathrm{tN}}\right) \leq \frac{\mathrm{c}_{0}}{\mathrm{t}^{\mathrm{p}}} \int_{\mathrm{t} / \mathrm{c}_{0}}^{\infty} \mathrm{v}\{\mathrm{f}>\mathrm{s}\} \mathrm{s}^{\mathrm{p}-1} \mathrm{ds}$.

Let now $\mathrm{N} \rightarrow \infty$. We use the same notation for the case $\mathrm{p}=1$ as above. Since now $u\left(Q_{j}\right) /\left|Q_{j}\right| \leqslant \inf Q_{j} v$ we get

$\mathrm{u}\left(\mathrm{E}_{\mathrm{tN}}\right) \leq \frac{\mathrm{c}}{\mathrm{t}} \sum \frac{\mathrm{u}\left(\mathrm{Q}_{\mathrm{j}}\right)}{\left|\mathrm{Q}_{\mathrm{j}}\right|} \int_{\mathrm{Q}_{\mathrm{j}}} \mathrm{f}^{\mathrm{t}}$

$\leq \frac{c}{t} \sum \int_{Q_{j}} f^{t} v \leq \frac{c}{t} \int_{R^{n}} f \chi_{A_{t}} v$.

Proceed now as in the case $1<\mathrm{p}<\infty$.

$(4) \rightarrow(5)$. For the case $p=1$ we fix a cube $Q_{0}$ and let $\mathrm{f}=\chi_{\mathrm{Q}}$, where $\mathrm{Q}$ is an arbitrary subcube of $\mathrm{Q}_{0}$. Then

$\mathrm{Q}_{0} \subset\left\{\mathrm{Mf} \geq \frac{1}{\left|\mathrm{Q}_{0}\right|} \int_{\mathrm{Q}} \mathrm{f}=\frac{|\mathrm{Q}|}{\left|\mathrm{Q}_{0}\right|} \equiv \mathrm{t}\right\}$.

Thus $\quad \mathrm{u}\left(\mathrm{Q}_{0}\right) \leq \mathrm{c}_{0}\left(\left|\mathrm{Q}_{0}\right| /|\mathrm{Q}|\right) \mathrm{v}(\mathrm{Q}), \quad$ and $\quad$ thus $\left.\mathrm{u}\left(\mathrm{Q}_{0}\right) / \mid \mathrm{Q}_{0}\right) \mid \leq \mathrm{c}_{0} \inf _{\mathrm{Q}_{0}} \mathrm{v}$.

If $1<\mathrm{p}<\infty$ we take the usual test function $\mathrm{f}=\chi_{\mathrm{Q}^{\mathrm{v}^{1}}} \mathrm{p}^{\prime}$ with $\mathrm{t}=\frac{1}{|\mathrm{Q}|} \int_{\mathrm{Q}} \mathrm{f}$. Then

$u(Q) \leq c_{0} \frac{|Q|^{p}}{\left(\int_{Q} f\right)^{p}} \int_{t / c_{0}}^{\infty} v\{f>s\} s^{p-1} d s$

$\leq \mathrm{c}_{0} \frac{|\mathrm{Q}|^{\mathrm{p}}}{\left(\int_{\mathrm{Q}} \mathrm{f}\right)^{\mathrm{p}}} \int_{\mathrm{Q}} \mathrm{f}^{\mathrm{p}}{ }_{\mathrm{v}}$

$=\mathrm{c}_{0}|\mathrm{Q}|^{\mathrm{p}}\left(\int_{\mathrm{Q}} \mathrm{v}^{1-\mathrm{p}^{\prime}}\right)^{1-\mathrm{p}}$,

and the $A_{p}$-condition follows.

3. PROOF OF THEOREM 1. (3) $\rightarrow$ (2).

$\int_{R^{n}} \Phi\left[\operatorname{Mf}(x)^{\mathrm{p}}\right] \mathrm{u}(\mathrm{x}) \mathrm{dx}=\int_{0}^{\infty} \mathrm{u}\left\{\mathrm{Mf}^{\mathrm{p}}>\mathrm{t}\right\} \mathrm{a}(\mathrm{t}) \mathrm{dt}=$

$\int_{0}^{\infty} \mathrm{u}\left\{\mathrm{Mf}>\mathrm{t}^{1 / \mathrm{p}}\right\} \mathrm{a}(\mathrm{t}) \mathrm{dt} \leq \mathrm{c}_{0} \int_{0}^{\infty} \frac{1}{\mathrm{t}} \int_{\mathrm{t}}^{\infty} \mathrm{t}^{1 / \mathrm{p}_{0}} \mathrm{v}\{\mathrm{f}>\mathrm{s}\} \mathrm{s}^{\mathrm{p}-1} \mathrm{dsa}(\mathrm{t}) \mathrm{dt}=$

$\mathrm{c}_{0} \int_{0}^{\infty} \int_{0}^{\left(\mathrm{c}_{0} \mathrm{~s}^{\mathrm{p}}\right.} \frac{\mathrm{a}(\mathrm{t})}{\mathrm{t}} \mathrm{v}\{\mathrm{f}>\mathrm{s}\} \mathrm{s}^{\mathrm{p}-1} \mathrm{dtds} \leq$

$\mathrm{c}_{0} \mathrm{c}^{\prime} \int_{0}^{\infty} \mathrm{b}\left(\mathrm{c}^{\prime \prime}\left(\mathrm{c}_{0} \mathrm{~s}\right)^{\mathrm{p}}\right) \mathrm{v}\{\mathrm{f}>\mathrm{s}\} \mathrm{s}^{\mathrm{p}-1} \mathrm{ds}=$

$\frac{c_{0} c^{\prime}}{p} \int_{0}^{\infty} b\left(c_{*} t\right) v\left\{f f^{p}>t\right\} d t \leq c_{1} \int_{R^{n}} \Psi\left[c_{2} f(x)^{p}\right] v(x) d x$. 
It is clear that the constants $c_{1}$ and $c_{2}$ have the desired properties.

$(2) \rightarrow(3)$. We assume that

$\mathrm{L} \equiv \int_{0}^{\infty} \mathrm{u}\left\{\mathrm{Mf} \mathrm{f}^{\mathrm{p}}>\mathrm{t}\right\} \mathrm{a}(\mathrm{t}) \mathrm{dt} \leq \mathrm{c}_{1} \int_{0}^{\infty} \mathrm{v}\left\{\mathrm{c}_{2} \mathrm{f}^{\mathrm{p}}>\mathrm{t}\right\} \mathrm{b}(\mathrm{t}) \mathrm{dt} \equiv \mathrm{R}$.

Fix $0<\lambda_{0}<\infty$ and let

$\mathrm{a}(\mathrm{t})=1 \mathrm{~h} \chi_{\left[\lambda_{0}, \lambda_{0}+\mathrm{h}\right]}(\mathrm{t})$.

Set

$b(t)=\int_{0}^{t} a(s) s d s=0,0 \leq t \leq \lambda_{0} 1 h \log \left(t / \lambda_{0}\right), \lambda_{0}<t \leq \lambda_{0}+h 1 h$

$\log \lambda_{0}+h \lambda_{0}, t>\lambda_{0}+h$.

With this choice $\Phi$ and $\Psi$ are $(1,1)$-related independent of $\mathrm{h}$ and $\lambda_{0}$ and hence $c_{1}$ and $c_{2}$ do not depend on $\mathrm{h}$ or $\lambda_{0}$. Then

$\mathrm{L}=\operatorname{lh} \int_{\lambda_{0}}^{\lambda_{0}+\mathrm{h}} \mathrm{u}\left\{\mathrm{Mf}^{\mathrm{p}}>\mathrm{t}\right\} \mathrm{dt} \rightarrow \mathrm{u}\left\{\mathrm{Mf}^{\mathrm{p}}>\lambda_{0}\right\}$

as $\mathrm{h} \rightarrow 0$. The right side $\mathrm{R}$ is

$R=c_{1} h \int_{\lambda_{0}}^{\lambda_{0}+h} v\left\{c^{2} f^{p}>t\right\} \log \left(t / \lambda_{0}\right) d t+c_{1} h \log \lambda_{0}+h \lambda_{0}$

$\int_{\lambda_{0}+h}^{\infty} v\left\{c_{2} f^{p}>t\right\} d t=I_{1}(h)+I_{2}(h)$.

We see that $\mathrm{I}_{1}(\mathrm{~h}) \rightarrow 0$ as $\mathrm{h} \rightarrow 0$ and

$\mathrm{I}_{2}(\mathrm{~h}) \rightarrow \mathrm{c}_{1} \lambda_{0} \int_{\lambda_{0}}^{\infty} \mathrm{v}\left\{\mathrm{c}_{2} \mathrm{f}^{\mathrm{p}}>\mathrm{t}\right\} \mathrm{dt}=\mathrm{c}_{1} \mathrm{c}_{2} \lambda_{0} \int_{\lambda_{0} / \mathrm{c}_{2}}^{\infty} \mathrm{v}\left\{\mathrm{f}^{\mathrm{p}}>\mathrm{t}\right\} \mathrm{dt}$.

Since $\lambda_{0}$ was arbitrary we get for some constant $c_{0}>1$

$\mathrm{u}\left\{\mathrm{Mf}^{\mathrm{p}}>\lambda\right\} \leq \mathrm{c}_{0} \lambda \int_{\lambda / \mathrm{c}_{0}}^{\infty} \mathrm{v}\left\{\mathrm{f}^{\mathrm{p}}>\mathrm{t}\right\} \mathrm{dt}$. get

We now make the substitution $\lambda=\mathrm{s}^{\mathrm{p}}$ and then $\mathrm{t} \rightarrow \mathrm{t}^{\mathrm{p}}$ to

$\mathrm{u}\{\mathrm{Mf}>\mathrm{s}\} \leq \mathrm{c}_{0} \mathrm{~s}^{\mathrm{p}} \int_{\mathrm{s} / \mathrm{c}_{0}}^{\infty} \mathrm{v}\{\mathrm{f}>\mathrm{t}\} \mathrm{t}^{\mathrm{p}-1} \mathrm{dt}$

By Theorem 2 this is the same as saying $(\mathrm{u}, \mathrm{v}) \in \mathrm{A}_{\mathrm{p}}$.

Remark. Theorem 1 is not true with $\mathbf{M}$ replaced by a singular integral operator $\mathrm{T}$. If it were true, then the argument as on the previous page shows that

$\mathrm{u}\{|\mathrm{Tf}|>\mathrm{s}\} \leq \mathrm{c}_{0} \mathrm{~s}^{\mathrm{p}} \int_{\mathrm{s} / \mathrm{c}_{0}}^{\infty} \mathrm{v}\{\mathrm{f}>\mathrm{t}\} \mathrm{t}^{\mathrm{p}-1} \mathrm{dt}$,

and hence for $\mathrm{s}>\mathrm{c}_{0}|\mathfrak{f}|_{b_{0}, \mathrm{v}}, \mathrm{u}\{|\mathrm{Tf}|>\mathrm{s}\}=0$ and $\mid \mathrm{Tf} \|_{\mathrm{b}_{0}, \mathrm{u}}<\infty$. But $\mathrm{T}$ is not of type $(\infty, \infty)[10]$.

\section{A CONVERSE}

For a given a, $\mathrm{b}: \mathrm{R}_{+} \rightarrow \mathrm{R}_{+}$and $\Phi(\mathrm{t})=\int_{0}^{\mathrm{t}} \mathrm{a}(\mathrm{s}) \mathrm{ds}, \Psi(\mathrm{t})=\int_{0}^{\mathrm{t}} \mathrm{b}(\mathrm{s}) \mathrm{ds}$ we wish to examine when (2) of Theorem 1 implies that $\int_{0}^{\mathrm{s}} \mathrm{a}(\mathrm{t}) \mathrm{tdt} \leq \mathrm{c}^{\prime} \mathrm{b}\left(\mathrm{c}^{\prime \prime} \mathrm{s}\right), 0<\mathrm{s}<\infty$.

Since this condition is independent of $(u, v) \in A_{p}$, we are allowed to take any $(u, v) \in A_{p}$, in particular $u=v=1$, the Lebesgue measure case, or $\mathrm{u}=\mathrm{v}$ in $\mathrm{RH}_{\infty}$. We prefer the second alternative since it is based on an extension of the reverse weak type inequality. We say that $\mathrm{u} \in \mathrm{RH}_{\infty}$ if for every cube $Q, \underset{Q}{\sup u(x) \leq c 1|Q| \int_{Q} u}$. The inf of all such c's is called the $\mathrm{RH}_{\infty}$-constant of $\mathrm{u}$. This class was studied in [2] and plays roughly the same role among the reverse Hölder classes $\mathrm{RH}_{\mathrm{r}}, \mathrm{r} \rightarrow \infty$, as $\mathrm{A}_{1}$ does among $\mathrm{A}_{\mathrm{p}}, \mathrm{p} \backslash 1$. Typical examples of $\mathrm{RH}_{\infty}$-weights in $\mathrm{R}_{+}$are $\mathrm{u}(\mathrm{x})=\mathrm{x}^{\alpha}, \alpha>0$.

Theorem 3 Let $\mathrm{u} \in \mathrm{RH}_{\infty}$. Then there are constants $0<c_{1}, c^{\prime}<\infty$ such that for all $\mathrm{f}: \mathrm{R}^{\mathrm{n}} \rightarrow \mathrm{R}_{+}$and $0<\mathrm{t}<\infty$

$1 t \int_{\{f>t\}} f(x) u(x) d x \leq c_{1} u\left\{\right.$ Mf $\left.>c^{\prime} t\right\}$,

where $1 / \mathrm{c}^{\prime}=\mathrm{c}_{*}$ is the $\mathrm{RH}_{\infty}$-constant of $\mathrm{u}$.

Proof. Since $\mathrm{u}(\mathrm{x}) \mathrm{dx}$ is a doubling measure [3], we have available the Calderon-Zygmund decomposition at height $t$ and this gives us disjoint cubes $\left\{\mathrm{Q}_{\mathrm{k}}\right\}$ such that

$$
\mathrm{t} \leq \mathrm{lu}\left(\mathrm{Q}_{\mathrm{k}}\right) \int_{\mathrm{Q}_{\mathrm{k}}} \mathrm{fu} \leq \mathrm{ct}
$$

$\mathrm{f}(\mathrm{x}) \leq \mathrm{t}$, on $\mathrm{R}^{\mathrm{n}} \cup \cup \mathrm{Q}_{\mathrm{k}}$.

Then

$1 \mathrm{t} \int_{\{\mathrm{f}>\mathrm{t}\}} \mathrm{fu} \leq 1 \mathrm{t} \sum \int_{\mathrm{Q}_{\mathrm{k}}} \mathrm{fu} \leq \mathrm{c} \sum \mathrm{u}\left(\mathrm{Q}_{\mathrm{k}}\right)=\mathrm{cu}\left(\cup \mathrm{Q}_{\mathrm{k}}\right) \leq \mathrm{cu}\left\{\mathrm{M}_{\mathrm{u}} \mathrm{f}>\mathrm{t}\right\}$,

where $M_{u} f(x)=\sup _{x \in Q} 1 u(Q) \int_{Q} f u$. Since $u \in R H_{\infty}$

$1 \mathrm{u}(\mathrm{Q}) \int_{\mathrm{Q}} \mathrm{fu} \leq \operatorname{supuu}_{\mathrm{Q}}(\mathrm{Q}) /|\mathrm{Q}| 1|\mathrm{Q}| \int_{\mathrm{Q}} \mathrm{f} \leq \mathrm{c} * \mathrm{Mf}(\mathrm{x})$,

if $x \in Q$. Hence $m_{u} f(x) \leq c * M f(x)$ and the proof is complete.

Defintion. (1) $b: R_{+} \rightarrow R_{+}$is quasi-increasing (qi) if there is a constant $0<\mathrm{c}_{0}<\infty$ such that $\mathrm{t}^{\prime} \leq \mathrm{t}^{\prime \prime}$ implies $\mathrm{b}\left(\mathrm{t}^{\prime}\right) \leq \mathrm{c}_{0} \mathrm{~b}\left(\mathrm{c}_{0} \mathrm{t}^{\prime \prime}\right)$

(2) A measure $\mu$ on $R_{+}$is weakly doubling if there is a constant $0<\mathrm{c}<\infty$ such that $\mu([0,2 \mathrm{~d}]) \leq \mathrm{c} \mu([\mathrm{d}, 2 \mathrm{~d}]), 0<\mathrm{d}<\infty$.

If a measure is doubling, it is also weakly doubling. The converse is not true as the measure $d \mu=e^{x} d x$ shows. In fact if 
$\mathrm{f}: \mathrm{R}_{+} \rightarrow \mathrm{R}_{+}$is nondecreasing, then $\mathrm{d} \mu=\mathrm{f}(\mathrm{x}) \mathrm{dx}$ is weakly doubling. The measure $\mathrm{d} \mu \mathrm{dx} /(1+\mathrm{x})$ is not weakly doubling.

Theorem 4 Assume that $b(t)$ is qi and assume that for some $\mathrm{n}$ and $\mathrm{u}_{0} \in \mathrm{RH}_{\infty}\left(\mathrm{R}^{\mathrm{n}}\right)$ we have

$\int_{\mathrm{R}^{\mathrm{n}}} \Phi\left(\mathrm{Mf}^{\mathrm{p}}\right) \mathrm{u}_{0} \leq \mathrm{c}_{1} \int_{\mathrm{R}^{\mathrm{n}}} \Psi\left(\mathrm{c}_{2} \mathrm{f}^{\mathrm{p}}\right) \mathrm{u}_{0}$

Then

$\int_{0}^{\mathrm{s}} \mathrm{a}(\mathrm{t}) \mathrm{tdt} \leq \mathrm{c}^{\prime} \mathrm{b}\left(\mathrm{c}^{\prime \prime} \mathrm{s}\right), 0<\mathrm{s}<\infty$

holds if $\mathrm{p}=1$, and if $1<\mathrm{p}<\infty$ it holds under the additional assumption that the measure $\mathrm{d} \mu=\mathrm{a}(\mathrm{t}) \mathrm{tdt}$ is weakly doubling.

Proof. In distributional form the integral inequality is

$\mathrm{L} \equiv \int_{0}^{\infty} \mathrm{u}_{0}\left\{\mathrm{Mf}^{\mathrm{p}}>\mathrm{t}\right\} \mathrm{a}(\mathrm{t}) \mathrm{dt} \leq \mathrm{c}_{1} \int_{0}^{\infty} \mathrm{u}_{0}\left\{\mathrm{c}_{2} \mathrm{f}^{\mathrm{p}}>\mathrm{t}\right\} \mathrm{b}(\mathrm{t}) \mathrm{dt} \equiv \mathrm{R}$.

The constants $c_{1}, c_{2}, \ldots$ appearing below only depend upon the constants in the overall hypothesis. By Lemma 3

$L \geq c_{3} \int_{0}^{\infty} a(t) t^{1 / p} \int_{\left\{f>c_{4} t^{1 / p}\right\}} f(x) u_{0}(x) d x d t$.

We apply this to the test functions $\mathrm{f}(\mathrm{x})=\mathrm{r} \chi_{\mathrm{Q}}(\mathrm{x}), 0<\mathrm{r}<\infty$, $\mathrm{Q}=[0,1]^{\mathrm{n}}$ and get

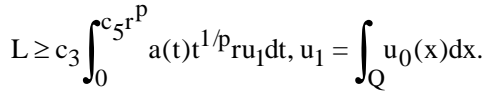

The right side $\mathrm{R}=\int_{0}^{\mathrm{c}} 6^{\mathrm{r}^{\mathrm{p}}} \mathrm{u}_{1} \mathrm{~b}(\mathrm{t}) \mathrm{dt}$. Hence

$c_{3} \int_{0}^{c_{5} 5^{r^{p}}} a(t) t^{1 / p} d t \leq c_{1} \int_{0}^{c_{6} 6^{r^{p}}} b(t) d t$.

With $\mathrm{s}=\mathrm{c}_{5} \mathrm{r}^{\mathrm{p}}$ this becomes

$\mathrm{c}_{7} \mathrm{~s}^{1 / \mathrm{p}} \int_{0}^{\mathrm{s}} \mathrm{a}(\mathrm{t}) \mathrm{t}^{1 / \mathrm{p}} \mathrm{dt} \leq \mathrm{c}_{1} \int_{0}^{\mathrm{c}_{8} \mathrm{~s}} \mathrm{~b}(\mathrm{t}) \mathrm{dt} \leq \mathrm{c}_{0} \mathrm{sb}\left(\mathrm{c}_{10} \mathrm{~s}\right)$

since $b$ is quasi-increasing. The left side is

$\geq \mathrm{c}_{7} \mathrm{~s}^{1 / \mathrm{p}} \int_{\mathrm{s} / 2}^{\mathrm{s}} \mathrm{t}^{1 / \mathrm{p}^{\prime}} \mathrm{a}(\mathrm{t}) \mathrm{tdt} \geq \mathrm{c}_{11} \mathrm{~s} \int_{0}^{\mathrm{s}} \mathrm{a}(\mathrm{t}) \mathrm{tdt}$

by the weak type doubling condition, which clearly is not needed when $\mathrm{p}=1$.

Remark: 1 . The special case $\mathrm{p}=1$ and $\mathrm{u}_{0} \sim 1$ - the Lebesgue measure case - is Theorem 7 in [6].

2. The weak doubling hypothesis of the measure $\mathrm{d} \mu=\mathrm{a}(\mathrm{t}) \mathrm{tdt}$ cannot be omitted if $1<\mathrm{p}<\infty$. The classical norm inequality for $\mathrm{u} \in \mathrm{A}_{\mathrm{p}}$ is

$\int_{R}{ }^{n}{ }^{p} u \leq c \int_{R}{ }^{n}{ }^{p} u$

This is the $\Phi(\mathrm{t})=\Psi(\mathrm{t})=\mathrm{t}$ case, and $\mathrm{a}(\mathrm{t})=1$.

\section{EXTRAPOLATION}

As before $\mathrm{a}, \mathrm{b}: \mathrm{R}_{+} \rightarrow \mathrm{R}_{+}$and $\Phi(\mathrm{s})=\int_{0}^{\mathrm{s}} \mathrm{a}(\mathrm{t}) \mathrm{dt}, \Psi(\mathrm{s})=\int_{0}^{\mathrm{s}} \mathrm{b}(\mathrm{t}) \mathrm{dt}$. We wish to examine the relationship between the following statements.

I. There exists $0<\varepsilon<\mathrm{p}, 1 \leq \mathrm{p}<\infty$, such that for $(\mathrm{u}, \mathrm{v}) \in \mathrm{A}_{\mathrm{p}}$ we have

$\int_{R^{n}} \Phi\left(\mathrm{Mf}^{\mathrm{p}-\varepsilon}\right) \mathrm{u} \leq \mathrm{c}_{1} \int_{\mathrm{R}^{\mathrm{n}}} \Psi\left(\mathrm{c}_{2} \mathrm{f}^{\mathrm{p}-\varepsilon}\right) \mathrm{v}$.

II. There exists $\eta>0$ such that

$\int_{0}^{\mathrm{s}} \mathrm{a}(\mathrm{t}) \mathrm{t}^{1+\eta} \mathrm{dt} \leq \mathrm{c}^{\prime} \mathrm{b}\left(\mathrm{c}^{\prime \prime} \mathrm{s}\right) \mathrm{s}^{\eta}, 0<\mathrm{s}<\infty$.

The constants $\varepsilon, \eta$, and $\mathrm{p}$ are related by $\varepsilon=\eta p /(1+\eta)$ or $\eta=\varepsilon /(p-\varepsilon)$.

Theorem $5 \mathrm{II} \Rightarrow \mathrm{I}$, and, if $\mathrm{b}$ is quasi-increasing and $\mathrm{u}=\mathrm{v}=1$, the converse $\mathrm{I} \Rightarrow$ II holds if $\mathrm{p}=1$, and if $1<\mathrm{p}<\infty$ it holds if the measure $d \mu=a(t) / t^{1+\eta} d t$ is weakly doubling.

Proof. II $\Rightarrow$ I. Fix $1 \leq \mathrm{p}<\infty$ and let $\varepsilon=\eta \mathrm{p} /(1+\eta)$. If $\mathrm{q}=\mathrm{p}-\varepsilon$, then $\mathrm{p} / \mathrm{q}=1+\eta$. By Theorem 2

$$
\begin{aligned}
& \int_{R^{n}} \Phi\left(M f^{q}\right) u \leq c_{0} \int_{0}^{\infty} a(t) t^{1+\eta} \int_{t^{1 / q} / c_{0}}^{\infty} v\{f>s\} s^{p-1} d s d t=c_{0} \int_{0}^{\infty} \int_{0}^{\left(c_{0} s\right)^{q}} \\
& a(t) t^{1+\eta} d t v\{f>s\} s^{p-1} d s \leq c \int_{0}^{\infty} b\left[\left(c_{0}^{\prime} s\right)^{q}\right]\left(c_{0} s\right)^{q \eta} v\{f>s\} s^{p-1} d s=c \int_{0}^{\infty} \\
& b(\sigma) \sigma^{\eta} v\left\{f>\sigma^{1 / q}\right\} \sigma^{(p-1) / q} \sigma^{1 / q-1} d \sigma=c \int_{0}^{\infty} v\left\{f>\sigma^{1 / q}\right\} b(\sigma) d \sigma=c_{1} \\
& \int_{R^{n}} \Psi\left(c_{2} f^{q}\right) v .
\end{aligned}
$$

$\mathrm{I} \Rightarrow \mathrm{II}$. First let $\mathrm{p}=1$ and $\mathrm{u}=\mathrm{v}=1$. If $\mathrm{q}=1-\varepsilon$, then the statement $\mathrm{I}$ in distributional form is

$\mathrm{L}=\int_{0}^{\infty}\left|\left\{\mathrm{Mf}>\mathrm{t}^{1 / \mathrm{q}}\right\}\right| \mathrm{a}(\mathrm{t}) \mathrm{dt} \leq \mathrm{c}_{1} \int_{0}^{\infty}\left|\left\{\mathrm{f}>\mathrm{c}_{3} \mathrm{t}^{1 / \mathrm{q}}\right\}\right| \mathrm{b}(\mathrm{t}) \mathrm{dt}=\mathrm{R}$.

By Lemma 3,

$L \geq c_{4} \int_{0}^{\infty} a(t) t^{1 / q} \int_{\left\{f>c_{5} t^{1 / q}\right\}} f(x) d x d t$.

We apply this to the test functions $f(x)=r \chi_{[0,1]}(x)$, $0<\mathrm{r}<\infty$. Then

$L \geq c_{4} \int_{0}^{c} 6^{r^{\mathrm{q}}} r a(t) t^{1 / q} d t, R=\int_{0}^{c_{7} r^{\mathrm{q}}} b(t) d t \leq c_{8} r^{q} b\left(c_{9} r^{\mathrm{q}}\right)$,

because $b$ is quasi-increasing. Hence

$\int_{0}^{\mathrm{c}} 6^{\mathrm{r}^{\mathrm{q}}} \mathrm{a}(\mathrm{t}) \mathrm{t}^{1 / \mathrm{q}} \mathrm{dt} \leq \mathrm{c}_{10} \mathrm{r}^{\mathrm{q}-1} \mathrm{~b}\left(\mathrm{c}_{9} \mathrm{r}^{\mathrm{q}}\right)$.

Let $\mathrm{s}=\mathrm{c}_{6} \mathrm{r}^{\mathrm{q}}$ and $1 / \mathrm{q}=1+\eta$. Then $\eta=\varepsilon /(1-\varepsilon)$ and

$\int_{0}^{\mathrm{s}} \mathrm{a}(\mathrm{t}) \mathrm{t}^{1+\eta} \mathrm{dt} \leq \mathrm{c}_{11^{\mathrm{s}}} \mathrm{s}^{(\mathrm{q}-1) / \mathrm{q}} \mathrm{b}\left(\mathrm{c}_{12} \mathrm{~s}\right)$, 
and $(\mathrm{q}-1) / \mathrm{q}=-\eta$.

The case $1<\mathrm{p}<\infty$ with $\mathrm{q}=\mathrm{p}-\varepsilon$, and $\mathrm{u}=\mathrm{v}=1$ follows the same steps as above and we get

$\int_{0}^{\mathrm{s}} \frac{\mathrm{a}(\mathrm{t})}{\mathrm{t}^{1 / \mathrm{q}}} \mathrm{dt} \leq \mathrm{c}_{11} \mathrm{~s}^{(\mathrm{q}-1) / \mathrm{q}} \mathrm{b}\left(\mathrm{c}_{12} \mathrm{~s}\right)$.

We use now the weak doubling condition and get

$\int_{0}^{\mathrm{s}} \frac{\mathrm{a}(\mathrm{t})}{\mathrm{t}^{1 / \mathrm{q}}} \mathrm{dt}=\int_{0}^{\mathrm{s}} \frac{\mathrm{a}(\mathrm{t}) \mathrm{t}^{1+\eta-1 / \mathrm{q}}}{\mathrm{t}^{1+\eta}} \geq \mathrm{c}_{13^{5}} \mathrm{~s}^{1+\eta-1 / \mathrm{q}} \int_{\mathrm{s} / 2}^{\mathrm{s}} \frac{\mathrm{a}(\mathrm{t})}{\mathrm{t}^{1+\eta}} \mathrm{dt} \geq \mathrm{c}_{14} \mathrm{~s}^{1+\eta-1 / \mathrm{q}} \int_{0}^{\mathrm{s}} \frac{\mathrm{a}(\mathrm{t})}{\mathrm{t}^{1+\eta}} \mathrm{dt}$.

Hence

$\int_{0}^{\mathrm{s}} \frac{\mathrm{a}(\mathrm{t})}{\mathrm{t}^{1+\eta}} \mathrm{dt} \leq \mathrm{c}_{15} \mathrm{~b}\left(\mathrm{c}_{12} \mathrm{~s}\right) / \mathrm{s}^{\eta}$

The result that we discuss now essentially says that, in the presence of condition II, extrapolation for $(u, v)$ is the same as $(\mathrm{u}, \mathrm{v}) \in \mathrm{A}_{\mathrm{p}}$.

Theorem 6 Let $1 \leq \mathrm{p}<\infty, \eta \geq 0, \varepsilon=\eta \mathrm{p} /(1+\eta)$, and

$\int_{0}^{\mathrm{s}} \frac{\mathrm{a}(\mathrm{t})}{\mathrm{t}^{1+\eta}} \mathrm{dt} \leq \frac{\mathrm{c}^{\prime} \mathrm{b}\left(\mathrm{c}^{\prime \prime} \mathrm{s}\right)}{\mathrm{s}^{\eta}}, 0<\mathrm{s}<\infty$.

Then the following statements are equivalent.

$\int_{\mathrm{R}^{\mathrm{n}}} \Phi\left(\mathrm{Mf}^{\mathrm{p}-\varepsilon}\right) \mathrm{u} \leq \mathrm{c}_{1} \int_{\mathrm{R}^{\mathrm{n}}} \Psi\left(\mathrm{c}_{2} \mathrm{f}^{\mathrm{p}-\varepsilon}\right) \mathrm{v}$,

where $\mathrm{c}_{1}, \mathrm{c}_{2}$ depend only upon $\mathrm{c}^{\prime}, \mathrm{c}^{\prime \prime}$ and $\mathrm{p}$.

(2) We have $(u, v) \in A_{p}$.

Remark: Theorem 1 is the special case $\eta=0$.

Proof. (2) $\Rightarrow(1)$. This is $\mathrm{II} \Rightarrow \mathrm{I}$ of Theorem 5. (1) $\Rightarrow(2)$. We proceed as in the proof of Theorem 1 and let $\mathrm{a}(\mathrm{t})=\frac{1}{\mathrm{~h}} \chi_{[\lambda, \lambda+\mathrm{h}]}(\mathrm{t}), \lambda>0, \mathrm{~h}>0$.

We let $b(s)=s^{\eta} \int_{0}^{s} \frac{a(t)}{t^{1+\eta}} d t$. We may assume that $\eta>0$ since the case $\eta=0$ is Theorem 1 . Then

$\mathrm{b}(\mathrm{s})=0,0 \leq \mathrm{s} \leq \lambda \frac{(\mathrm{s} / \lambda)^{\eta}-1}{\mathrm{~h} \eta}, \lambda \leq \mathrm{s} \leq \lambda+\mathrm{h} \frac{(\mathrm{s} / \lambda)^{\eta}-(\mathrm{s} /(\lambda+\mathrm{h}))^{\eta}}{\mathrm{h} \eta}, \mathrm{s} \geq \lambda+\mathrm{h}$.

Our hypothesis in distributional form is $\mathrm{L}_{\mathrm{h}} \equiv \int_{0}^{\infty} \mathrm{u}\left\{\mathrm{Mf}^{\mathrm{p}-\varepsilon}>\mathrm{t}\right\} \mathrm{a}(\mathrm{t}) \mathrm{dt} \leq \mathrm{c}_{1} \int_{\lambda}^{\infty} \mathrm{v}\left\{\mathrm{c}_{2} \mathrm{f}^{\mathrm{p}-\varepsilon}>\mathrm{t}\right\} \mathrm{b}(\mathrm{t}) \mathrm{dt} \equiv \mathrm{R}_{\mathrm{h}}$.

First

$\mathrm{L}_{\mathrm{h}}=\frac{1}{\mathrm{~h}} \int_{\lambda}^{\lambda+\mathrm{h}} \mathrm{u}\left\{\mathrm{Mf}^{\mathrm{p}-\varepsilon}>\mathrm{t}\right\} \mathrm{dt} \rightarrow \mathrm{u}\left\{\mathrm{Mf}^{\mathrm{p}-\varepsilon}>\lambda\right\}$

as $h \rightarrow 0$. The right side $R_{h}$ splits into two integrals

$\mathrm{R}_{\mathrm{h}}=\mathrm{c}_{1}\left(\int_{\lambda}^{\lambda+\mathrm{h}}+\int_{\lambda+\mathrm{h}}^{\infty}\right)=\mathrm{I}_{1}+\mathrm{I}_{2}$.
$I_{1}$ is easily disposed of

$\mathrm{I}_{1}=\mathrm{c}_{1} \int_{\lambda}^{\lambda+\mathrm{h}} \frac{(\mathrm{t} / \lambda)^{\eta}-1}{\mathrm{~h} \eta} \mathrm{v}\left\{\mathrm{c}_{2} \mathrm{f}^{\mathrm{p}-\varepsilon}>\mathrm{t}\right\} \mathrm{dt} \rightarrow 0$,

as $h \rightarrow 0$. Next

$\mathrm{I}_{2}=\mathrm{c}_{1} \frac{\lambda^{-\eta}-(\lambda+\mathrm{h})^{-\eta}}{\mathrm{h} \eta} \int_{\lambda+\mathrm{h}}^{\infty} \mathrm{v}\left\{\mathrm{c}_{2} \mathrm{f}^{\mathrm{p}-\varepsilon}>\mathrm{t}\right\} \mathrm{t}^{\eta} \mathrm{dt} \rightarrow \frac{\mathrm{c}_{1}}{\lambda^{\eta+1}} \int_{\lambda}^{\infty} \mathrm{v}\left\{\mathrm{c}_{2} \mathrm{f}^{\mathrm{p}-\varepsilon}>\mathrm{t}\right\} \mathrm{t}^{\eta} \mathrm{dt}$,

as $\mathrm{h} \rightarrow 0$. The substitution $\tau=\mathrm{t}^{\eta+1}$ gives

$\mathrm{I}_{2} \rightarrow \frac{\mathrm{c}_{3}}{\lambda^{\eta+1}} \int_{\lambda}^{\infty} \eta^{\eta+1} \mathrm{v}\left\{\mathrm{c}_{2} \mathrm{f}^{\mathrm{p}-\varepsilon}>\tau^{1 /(\eta+1)}\right\} \mathrm{d} \tau$,

and since $(p-\varepsilon)(\eta+1)=p(\eta+1)-p \eta=p$,

$\mathrm{I}_{2} \rightarrow \frac{\mathrm{c}_{3}}{\lambda^{\eta+1}} \int_{\lambda}^{\infty}{ }^{\eta+1} \mathrm{v}\left\{\mathrm{c}_{4} \mathrm{f}^{\mathrm{p}}>\tau\right\} \mathrm{d} \tau$

Hence for some constant $c_{0}>1$

$\mathrm{u}\left\{\mathrm{Mf}^{\mathrm{p}-\varepsilon}>\lambda\right\} \leq \frac{\mathrm{c}_{0}}{\lambda^{\eta+1}} \int_{\lambda^{\eta+1} / \mathrm{c}_{0}}^{\infty} \mathrm{v}\left\{\mathrm{f}^{\mathrm{p}}>\mathrm{t}\right\} \mathrm{dt}$.

With $\lambda=\sigma^{\mathrm{p}-\varepsilon}$ we get

$\mathrm{u}\{$ Mf $>\sigma\} \leq \frac{\mathrm{c}_{0}}{\sigma^{\mathrm{p}}} \int_{\sigma / \mathrm{c}_{0}}^{\infty} \mathrm{v}\left\{\mathrm{f}^{\mathrm{p}}>\mathrm{t}\right\} \mathrm{dt}=\frac{\mathrm{c}_{0^{\prime}}}{\sigma^{\mathrm{p}}} \int_{\sigma / \mathrm{c}_{0^{\prime}}}^{\infty} \mathrm{v}\{\mathrm{f}>\mathrm{t}\} \mathrm{t}^{\mathrm{p}-1} \mathrm{dt}$.

This shows that $(\mathrm{u}, \mathrm{v}) \in \mathrm{A}_{\mathrm{p}}$ by Theorem 2 .

Remark: The following observation may be of interest in connection with condition II: if $\int_{0}^{\mathrm{s}} \frac{\mathrm{a}(\mathrm{t})}{\mathrm{t}} \mathrm{dt} \leq \mathrm{c}_{0} \mathrm{a}(\mathrm{s})$, then there exists $\eta>0$ such that

$\int_{0}^{\mathrm{s}} \frac{\mathrm{a}(\mathrm{t})}{\mathrm{t}^{1+\eta}} \mathrm{dt} \leq \mathrm{c} \frac{\mathrm{a}(\mathrm{s})}{\mathrm{s}^{\eta}}$

and hence Theorem 5 about extrapolation applies.

Proof. By hypothesis

$\mathrm{L} \equiv \int_{0}^{\mathrm{s}_{1}} \frac{1}{\mathrm{~s}} \int_{0}^{\mathrm{s}} \frac{\mathrm{a}(\mathrm{t})}{\mathrm{t}} \mathrm{dtds} \leq \mathrm{c}_{0} \int_{0}^{\mathrm{s}_{1}} \leq \mathrm{c}_{0}^{2} \mathrm{a}\left(\mathrm{s}_{1}\right)$.

Also

$\mathrm{L}=\int_{0}^{\mathrm{s}_{1}} \int_{\mathrm{t}}^{\mathrm{s}_{1}} \frac{\mathrm{a}(\mathrm{t})}{\mathrm{ts}} \mathrm{dsdt}=\int_{0}^{\mathrm{s}_{1}} \frac{\mathrm{a}(\mathrm{t})}{\mathrm{t}} \log \frac{\mathrm{s}_{1}}{\mathrm{t}} \mathrm{dt} \leq \mathrm{c}_{0}^{2} \mathrm{a}\left(\mathrm{s}_{1}\right)$.

We repeat this argument and finally get

$\int_{0}^{\mathrm{s}} \frac{\mathrm{a}(\mathrm{t})}{\mathrm{t}} \frac{1}{\mathrm{j} !} \log \frac{\mathrm{s}}{\mathrm{t}} \mathrm{dt} \leq \mathrm{c}_{0}^{\mathrm{j}+1} \mathrm{a}(\mathrm{s})$.

Let $c_{1}>c_{0}$. Then

$\int_{0}^{\mathrm{s}} \frac{\mathrm{a}(\mathrm{t})}{\mathrm{t}} \sum \frac{1}{\mathrm{j} !} \frac{1}{\mathrm{c}_{1}^{\mathrm{j}}} \log \frac{\mathrm{s}}{\mathrm{t}} \mathrm{dt} \leq \mathrm{ca}(\mathrm{s})$,

and the sum $=(\mathrm{s} / \mathrm{t})^{\eta}$ with $\eta=1 / \mathrm{c}_{1}$. 


\section{ITERATED MAXIMAL OPERATOR. LET}

$M_{j} f(x)=\underbrace{M \circ M \circ \cdots \circ M}_{j-\text { times }} f(x)$.

The purpose of this section is to present some weighted integral inequalities involving $\mathrm{M}_{\mathrm{j}} \mathrm{f}$.

Theorem 7 Let $\mathrm{u} \in \mathrm{A}_{\mathrm{p}}, 1 \leq \mathrm{p}<\infty$, and assume that $a, b: R_{+} \rightarrow R_{+}$satisfy

$\int_{0}^{\mathrm{s}} \frac{\mathrm{a}(\mathrm{t})}{\mathrm{t}} \log ^{\mathrm{j}-1}(\mathrm{~s} / \mathrm{t}) \mathrm{dt} \leq \mathrm{c}^{\prime} \mathrm{b}\left(\mathrm{c}^{\prime \prime} \mathrm{s}\right)$.

Then, if $\Phi(\mathrm{t})=\int_{0}^{\mathrm{t}} \mathrm{a}(\mathrm{s}) \mathrm{d} \mathrm{s}, \Psi(\mathrm{t})=\int_{0}^{\mathrm{t}} \mathrm{b}(\mathrm{s}) \mathrm{ds}$,

$\int_{R^{n}} \Phi\left(M_{j} f^{p}\right) u \leq c_{j^{\prime}} \int_{R^{n}} \Psi\left(c_{j^{\prime \prime}} f^{p}\right) u$.

Proof. By Theorem 2,

$u\left\{M_{j} f>t\right\} \leq \frac{c_{o}}{t^{p}} \int_{t / c_{o}}^{\infty} u\left\{M_{j-1} f>s_{1}\right\} s_{1}^{p-1} d s_{1} \leq \frac{c_{0}^{2}}{t^{p}} \int_{t / c_{o}}^{\infty} \frac{s_{1}^{p-1}}{s_{1}^{p}} \int_{s_{1} / c_{o}}^{\infty}$

$u\left\{M_{j-2} f>s_{2}\right\} s_{2}^{p-1} d s_{2} d s_{1}=\frac{c_{o}^{2}}{t^{p}} \int_{t / c_{o}^{2}}^{\infty} \int_{t / c_{o}}^{c_{0} s_{2}} \frac{d s_{1}}{s_{1}} u\left\{M_{j-2} f>s_{2}\right\} s_{2}^{p-1} d s_{2}$

$=\frac{c_{o}^{2}}{t^{p}} \int_{t / c_{o}^{2}}^{\infty} \log \frac{c_{o}^{2} s_{2}}{t} u\left\{M_{j-2}>s_{2}\right\} s_{2}^{p-1} d s_{2} \leq \cdots \leq \frac{c_{o}^{j}}{(j-1) ! t^{p}} \int_{t / c_{0}^{j}}^{\infty}$

$\log ^{j-1} \frac{c_{o}^{j} s}{t} u\{f>s\} s^{p-1} d s$

The left side of the conclusion is

$\int_{0}^{\infty} u\left\{M_{j} f>t^{1 / p}\right\} a(t) d t \leq \frac{c_{o}^{j}}{(j-1) !} \int_{0}^{\infty} \frac{a(t)}{t} \int_{t^{1 / p} / c_{o}^{j}}^{\infty} \log ^{j-1} \frac{c_{o}^{j} s}{t^{1 / p}} u\{f>$

$s\} s^{p-1} d s d t=\frac{c_{o}^{j}}{(j-1) !} \int_{0}^{\infty} \frac{a(t)}{t} \int_{t / c_{o}^{j p}}^{\infty} \log ^{1-1}\left(c_{o}^{j}(\sigma / t)^{1 / p}\right) u\left\{f>\sigma^{1 / p}\right\} d \sigma d t=$

$\frac{\mathrm{cc}_{0}^{\mathrm{j}}}{(\mathrm{j}-1) !} \int_{0}^{\infty} \int_{0}^{\mathrm{c}_{\mathrm{o}}^{\mathrm{jp}} \sigma} \frac{\mathrm{a}(\mathrm{t})}{\mathrm{t}} \log ^{\mathrm{j}-1}\left(\mathrm{c}_{\mathrm{o}}^{\mathrm{jp}} \sigma / \mathrm{t}\right) \mathrm{dtu}\left\{\mathrm{f}>\sigma^{1 / \mathrm{p}}\right\} \mathrm{d} \sigma=\frac{\mathrm{cc}_{\mathrm{o}}^{\mathrm{j}}}{(\mathrm{j}-1) !} \int_{0}^{\infty}$

$\mathrm{b}\left(\mathrm{c}^{\prime \prime} \mathrm{c}_{\mathrm{o}}^{\mathrm{jp}} \sigma\right) \mathrm{u}\left\{\mathrm{f}>\sigma^{1 / \mathrm{p}}\right\} \mathrm{d} \sigma \leq \mathrm{c}_{\mathrm{j}^{\prime}} \int_{\mathrm{R}^{\mathrm{n}}} \Psi\left(\mathrm{c}_{\mathrm{j}^{\prime \prime}} \mathrm{f}^{\mathrm{p}}\right) \mathrm{u}$.

Remark: (1) The log term in the hypothesis of Theorem 7 can be omitted if $\mathrm{u} \sim 1$, the Lebesgue measure case and $1<\mathrm{p}<\infty$. The operator $\mathrm{M}_{\mathrm{j}} \mathrm{f}$ is weak (p,p) and $(\infty, \infty)$ and hence by $[11, \mathrm{p} .103]$

$\left|\left\{M_{j} f>t\right\}\right| \leq \frac{c_{j}}{t^{p}} \int_{t / c_{j}}^{\infty}|\{f>s\}| s^{p-1} d s$.

$>$ From this we get

$$
\begin{aligned}
& \int_{R^{n}} \Phi\left(M_{j} f(x)^{p}\right) d x=\int_{0}^{\infty}\left|\left\{M_{j} f>t^{1 / p}\right\}\right| a(t) d t \leq c_{j} \int_{0}^{\infty} \frac{a(t)}{t} \int_{t^{1 / p} / c_{j}}^{\infty} \\
& |\{f>s\}| s^{p-1} d s d t=c_{j} \int_{0}^{\infty} \int_{0}^{\left(c_{j} s\right)^{p}} \frac{a(t)}{t}|\{f>s\}| s^{p-1} d t d s=c_{1} \int_{0}^{\infty} b\left(c_{2} s^{p}\right) \\
& |\{f>s\}| s^{p-1} d s=c_{3} \int_{0}^{\infty} b\left(c_{2} t\right)\left|\left\{f^{p}>t\right\}\right| d t=c_{j^{\prime}} \int_{R^{n}} \Psi\left(c_{j^{\prime \prime}} f(x)^{p}\right) d x .
\end{aligned}
$$

(2) There is a converse to the above. If $b$ is qi, the integral inequality

$\int_{R^{n}} \Phi\left(M_{j} f(x)^{p}\right) d x \leq c_{j^{\prime}} \int_{R^{n}} \Psi\left(c_{j^{\prime \prime}} f(x)^{p}\right) d x$

implies

$\int_{0}^{\mathrm{s}} \frac{\mathrm{a}(\mathrm{t})}{\mathrm{t}} \mathrm{dt} \leq \mathrm{c}^{\prime} \mathrm{b}\left(\mathrm{c}^{\prime \prime} \mathrm{s}\right), 0<\mathrm{s}<\infty$,

if $\mathrm{p}=1$, and if $\mathrm{p}>1$ this holds if the measure $\mathrm{d} \mu=\frac{\mathrm{a}(\mathrm{t})}{\mathrm{t}} \mathrm{dt}$ is weakly doubling. This follows from

$\int_{\mathrm{R}^{\mathrm{n}}} \Phi\left(\operatorname{Mf}(\mathrm{x})^{\mathrm{p}}\right) \mathrm{dx} \leq \int_{\mathrm{R}^{\mathrm{n}}} \Phi\left(\mathrm{M}_{\mathrm{j}} \mathrm{f}(\mathrm{x})^{\mathrm{p}}\right) \mathrm{dx} \leq \mathrm{c}_{\mathrm{j}^{\prime}} \int_{\mathrm{R}^{\mathrm{n}}} \Psi\left(\mathrm{c}_{\mathrm{j}^{\prime \prime}} \mathrm{f}(\mathrm{x})^{\mathrm{p}}\right) \mathrm{dx}$,

and Theorem 4 applies.

\section{THE ITERATED MAX OPERATOR AND EXTRAPOLATION}

There is a connection between the behavior of $\mathrm{M}_{\mathrm{j}} \mathrm{f}$ and extrapolation [7-9]. The next two Theorems will explore this connection in our setting. Again let $a, b: R_{+} \rightarrow R_{+}$and let $\Phi(\mathrm{s})=\int_{0}^{\mathrm{s}} \mathrm{a}(\mathrm{t}) \mathrm{dt}, \Psi(\mathrm{s})=\int_{0}^{\mathrm{s}} \mathrm{b}(\mathrm{t}) \mathrm{dt}$ with $\mathrm{b}$ quasi-increasing.

Theorem 8 Let $1 \leq p<\infty$ and assume that for $j \in N$ $\int_{R} \Phi\left(M_{j} f(x)^{p}\right) d x \leq A^{j} \int_{R} \Psi\left(c_{2} f(x)^{p}\right) d x$,

with $c_{2}$ independent of $j$. Let $A<c_{*}<\infty$ and let $\eta=1 /\left(c_{*} * \mathrm{p}\right)$. If in the case $1<\mathrm{p}$ the measure $\mathrm{d} \mu=\frac{\mathrm{a}(\mathrm{t})}{\mathrm{t}^{1+\eta}} \mathrm{dt}$ is weakly doubling, then for $(u, v) \in A_{p}\left(R^{n}\right)$

$\int_{R^{n}} \Phi\left(\mathrm{Mf}^{\mathrm{p}-\varepsilon}\right) \mathrm{u} \leq \mathrm{c}_{1^{\prime}} \int_{\mathrm{R}^{\mathrm{n}}} \Psi\left(\mathrm{c}_{1^{\prime \prime}} \mathrm{f}^{\mathrm{p}-\varepsilon}\right) \mathrm{v}$,

where $\varepsilon=\eta p /(1+\eta)$.

Proof. Our goal is to prove

$\int_{0}^{\mathrm{s}} \frac{\mathrm{a}(\mathrm{t})}{\mathrm{t}^{1+\eta}} \mathrm{dt} \leq \frac{\mathrm{c}^{\prime} \mathrm{b}\left(\mathrm{c}^{\prime \prime} \mathrm{s}\right)}{\mathrm{s}^{\eta}}$

and then Theorem 5 gives us our conclusion.

In distributional form our hypothesis is

$\mathrm{L} \equiv \int_{0}^{\infty}\left|\left\{\mathrm{M}_{\mathrm{j}} \mathrm{f}>\mathrm{t}^{1 / \mathrm{p}}\right\}\right| \mathrm{a}(\mathrm{t}) \mathrm{dt} \leq \mathrm{A}^{\mathrm{j}} \int_{0}^{\infty}\left|\left\{\mathrm{f}>\left(\mathrm{t} / \mathrm{c}_{2}\right)^{1 / \mathrm{p}}\right\}\right| \mathrm{b}(\mathrm{t}) \mathrm{dt} \equiv \mathrm{R}$.

By Lemma 3

$L \geq c_{1} \int_{0}^{\infty} \frac{a(t)}{t^{1 / p}} \int_{\left\{M_{j-1} f>c_{3} t^{1 / p}\right\}} M_{j-1} f(x) d x d t$

with $c_{1}, c_{3}$ independent of $\mathrm{j}$. We apply this to the test functions $f(x)=r \chi_{[0,1]}(x), 0<r<\infty$. Then

$\mathrm{M}_{\mathrm{i}} \mathrm{f}(\mathrm{x})=\mathrm{r}\left\{\chi_{[0,1]}(\mathrm{x})+\frac{1}{\mathrm{x}} \phi_{\mathrm{i}-1}(\mathrm{x}) \chi_{[1, \infty)}(\mathrm{x})\right\}, \phi_{\mathrm{k}}(\mathrm{x})=\sum_{0}^{\mathrm{k}} \frac{\log ^{\mathrm{i}} \mathrm{x}}{\mathrm{i} !}$. 
Therefore the inner integral is

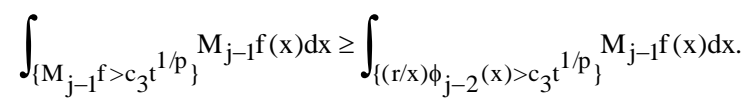

For $0<\mathrm{t}<\left(\mathrm{r} / \mathrm{c}_{3}\right)^{\mathrm{p}}$, the set $\left\{(\mathrm{r} / \mathrm{x}) \phi_{\mathrm{j}-2}(\mathrm{x})>\mathrm{c}_{3} \mathrm{t}^{1 / \mathrm{p}}\right\} \supset[1, \sigma(\mathrm{t}))$, where $\sigma(t)$ is defined by

$(\mathrm{r} / \sigma(\mathrm{t})) \phi_{\mathrm{j}-2}(\sigma(\mathrm{t}))=\mathrm{c}_{3} \mathrm{t}^{1 / \mathrm{p}}$.

Since $\phi_{\mathrm{j}-2}(\mathrm{x}) \geq 1$, we get $\sigma(\mathrm{t}) \geq \mathrm{r} /\left(\mathrm{c}_{3} \mathrm{t}^{1 / \mathrm{p}}\right)$. Hence

$\int_{\left\{M_{j-1}>c_{3} t^{1 / p}\right\}} M_{j-1} f(x) d x \geq \int_{1}^{r /\left(c_{3^{1}}{ }^{1 / p}\right)} \frac{r}{x(j-2) !} \log ^{j-2} x d x=\frac{r}{(j-1) !} \log ^{j-1} \frac{r}{c_{3} t^{1 / p}}=$ $\frac{r}{p^{j-1}(j-1) !} \log ^{j-1} \frac{r^{p}}{c_{3}^{p}}$.

Thus

$L \geq \frac{c_{1} r}{p^{j-1}(j-1) !} \int_{0}^{\left(r / c_{3}\right)^{p}} \frac{a(t)}{t^{1 / p}} \log \frac{j-1}{c_{3}} \frac{r^{p}}{c^{p_{t}}} d t$

Also

$\mathrm{R} \leq \mathrm{A}^{\mathrm{j}} \int_{0}^{\mathrm{c}_{4} \mathrm{r}^{\mathrm{p}}} \mathrm{b}(\mathrm{t}) \mathrm{dt} \leq \mathrm{A}^{\mathrm{j}} \mathrm{c}^{\prime} \mathrm{r}^{\mathrm{p}} \mathrm{b}\left(\mathrm{c}^{\prime \prime} \mathrm{r}^{\mathrm{p}}\right)$

since $b$ is quasi-increasing. Let $s=\left(r / c_{3}\right)^{p}$. Then

$\frac{\mathrm{c}_{5}}{\mathrm{p}^{\mathrm{j}-1}(\mathrm{j}-1) !} \mathrm{s}^{1 / \mathrm{p}} \int_{0}^{\mathrm{s}} \frac{\mathrm{a}(\mathrm{t})}{\mathrm{t}^{1 / \mathrm{p}}} \log ^{\mathrm{j}-1}(\mathrm{~s} / \mathrm{t}) \mathrm{dt} \leq \mathrm{A}^{\mathrm{j}} \mathrm{c}_{6} \mathrm{sb}\left(\mathrm{c}_{7} \mathrm{~s}\right)$.

Then

$\frac{\mathrm{c}_{5}}{\mathrm{c}_{*}} \mathrm{~s}^{1 / \mathrm{p}} \int_{0}^{\mathrm{s}} \frac{\mathrm{a}(\mathrm{t})}{\mathrm{t}^{1 / \mathrm{p}}} \sum \frac{1}{(\mathrm{j}-1) ! \mathrm{p}^{\mathrm{j}-1} \mathrm{c}_{*}^{\mathrm{j}-1}} \log { }^{\mathrm{j}-1}(\mathrm{~s} / \mathrm{t}) \mathrm{dt} \leq \mathrm{c}_{8} \mathrm{sb}\left(\mathrm{c}_{7} \mathrm{~s}\right)$,

since $c_{*}>A$. Since the sum inside the integral $=(s / t)^{\eta}$, we get

$\mathrm{L}_{1} \equiv \mathrm{c}_{9} \mathrm{~s}^{1 / \mathrm{p}} \int_{0}^{\mathrm{s}} \frac{\mathrm{a}(\mathrm{t})}{\mathrm{t}^{1 / \mathrm{p}}}(\mathrm{s} / \mathrm{t})^{\eta} \mathrm{dt} \leq \mathrm{c}_{8} \mathrm{sb}\left(\mathrm{c}_{7} \mathrm{~s}\right)$.

If $1=p$ we stop, and if $p>1$ we note that

$\mathrm{L}_{1} \geq \mathrm{c}_{10} \int_{\mathrm{s} / 2}^{\mathrm{s}} \frac{\mathrm{a}(\mathrm{t})}{\mathrm{t}}(\mathrm{s} / \mathrm{t})^{\eta} \mathrm{dt}$.

Finally, the weak doubling condition gives us

$\int_{0}^{\mathrm{s}} \frac{\mathrm{a}(\mathrm{t})}{\mathrm{t}^{1+\eta}} \mathrm{dt} \leq \frac{\mathrm{c}_{11} \mathrm{~b}\left(\mathrm{c}_{7} \mathrm{~s}\right)}{\mathrm{s}^{\eta}}$.

There is a converse to Theorem 8 which reads as follows.

Theorem 9 Let $1 \leq \mathrm{p}<\infty$ and assume that for some $\varepsilon>0$

$\int_{R^{n}} \Phi\left(M f^{p-\varepsilon}(x)\right) d x \leq c_{1} \int_{R^{n}} \Psi\left(c_{2} f^{p-\varepsilon}(x)\right) d x$.

If in case $\mathrm{p}>1$ the measure $\mathrm{d} \mu=\frac{\mathrm{a}(\mathrm{t})}{\mathrm{t}^{1+\eta}} \mathrm{dt}, \eta=\varepsilon /(\mathrm{p}-\varepsilon)$, is weakly doubling, then for $j \in N$ and $u \in A_{p}$

$\int_{R}{ }^{n} \Phi\left(M_{j} f^{p}\right) u \leq c_{j^{\prime}} \int_{R^{n}} \Psi\left(c_{j^{\prime \prime}} f^{p}\right) u$.
Proof. By Theorem 5, $\int_{0}^{\mathrm{s}} \frac{\mathrm{a}(\mathrm{t})}{\mathrm{t}^{1+\eta}} \mathrm{dt} \leq \frac{\mathrm{c}^{\prime} \mathrm{b}\left(\mathrm{c}^{\prime \prime} \mathrm{s}\right)}{\mathrm{s}^{\eta}}$. Then

$\int_{0}^{\mathrm{s}} \frac{\mathrm{a}(\mathrm{t})}{\mathrm{t}} \sum \frac{\eta^{\mathrm{j}-1}}{(\mathrm{j}-1) !} \log ^{\mathrm{j}-1}(\mathrm{~s} / \mathrm{t}) \mathrm{dt} \leq \mathrm{c}^{\prime} \mathrm{b}\left(\mathrm{c}^{\prime \prime} \mathrm{s}\right)$.

Thus for each $\mathrm{j} \in \mathrm{N}$

$\int_{0}^{\mathrm{s}} \frac{\mathrm{a}(\mathrm{t})}{\mathrm{t}} \log ^{\mathrm{j}-1}(\mathrm{~s} / \mathrm{t}) \mathrm{dt} \leq \mathrm{c}_{\mathrm{j}^{\prime}} \mathrm{b}\left(\mathrm{c}^{\prime \prime} \mathrm{s}\right)$.

Theorem 7 completes the proof.

\section{APPLICATIONS}

We give some examples of $\Phi$ and $\Psi$ which are $\left(\mathrm{c}^{\prime}, \mathrm{c}^{\prime \prime}\right)$ related and investigate the inplications of Theorem 1 . We will get some unusual and surprising integral inequalities.

I. If $(u, v) \in A_{p}$ for some $1 \leq p<\infty$, then

$\int_{R^{n}} M f^{r} u \leq c \int_{R} f^{r} v$,

for $\mathrm{p}<\mathrm{r}<\infty$.

Proof. This is well-known [4]. It also follows from Theorem 1 by taking $\Phi(t)=t^{\alpha}, \alpha>1$. An easy calculation shows that we can take $\Psi(\mathrm{t})=\mathrm{t}^{\alpha}$.

II. If $(u, v) \in A_{p}$ for some $1 \leq p<\infty$, then for $\alpha>1$

$\int_{R^{n}} \log ^{\alpha}\left(1+M f^{p}\right) u \leq c \int_{R^{n}} f^{p} \log ^{\alpha-1}\left(1+f^{p}\right) v$.

Proof. Let $\Phi(\mathrm{t})=\log ^{\alpha}(1+\mathrm{t})$. Then $\mathrm{a}(\mathrm{t})=\alpha \frac{\log ^{\alpha-1}(1+\mathrm{t})}{1+\mathrm{t}}$ and

$\int_{0}^{\mathrm{s}} \frac{\mathrm{a}(\mathrm{t})}{\mathrm{t}} \mathrm{dt}=\alpha \int_{0}^{\mathrm{s}} \frac{\log ^{\alpha-1}(1+\mathrm{t})}{\mathrm{t}(1+\mathrm{t})} \mathrm{dt} \leq \alpha \int_{0}^{\mathrm{s}} \frac{\log ^{\alpha-2}(1+\mathrm{t})}{1+\mathrm{t}} \mathrm{dt}=\frac{\alpha}{\alpha-1} \log ^{\alpha-1}(1+\mathrm{s})=\mathrm{b}(\mathrm{s})$.

Also

$\int_{0}^{\mathrm{t}} \mathrm{b}(\mathrm{s}) \mathrm{ds} \leq \frac{\alpha}{\alpha-1} \mathrm{t} \log ^{\alpha-1}(1+\mathrm{t}) \equiv \Psi(\mathrm{t})$.

The desired integral inequality follows from Theorem 1 , since $\log (1+\mathrm{cx}) \leq \mathrm{cog}(1+\mathrm{x})$ if $\mathrm{c} \geq 1$.

Remark: We cannot replace the right side by the more symmetric $\int_{R^{n}} \log ^{\alpha}\left(1+f^{p}\right) v$. As an example let $u=v=1$ and $\mathrm{n}=1 . \quad$ If $\quad \mathrm{f}(\mathrm{x})=\mathrm{r} \chi_{[0,1]}(\mathrm{x}), 0<\mathrm{r}<\infty, \quad$ then $\int_{R} \log ^{\alpha}\left(1+f^{p}\right)=\log ^{\alpha}\left(1+r^{p}\right)$. Since $\operatorname{Mf}(x) \geq r / x, x \geq 1$, we get $\int_{R} \log ^{\alpha}\left(1+M f^{\mathrm{p}}\right) \mathrm{dx} \geq \int_{1}^{\infty} \log ^{\alpha}\left(1+(\mathrm{r} / \mathrm{x})^{\mathrm{p}}\right) \mathrm{dx}$.

The integrand

$\log ^{\alpha}\left(1+(r / x)^{p}\right)=\left(\log \left(x^{p}+r^{p}\right)-\log x^{p}\right)^{\alpha} \geq\left(\frac{r^{p}}{x^{p}+r^{p}}\right)^{\alpha} \geq 1 / 2^{\alpha}$,

if $x \leq r$. Hence 
$\int_{R} \log ^{\alpha}\left(1+M f^{p}\right) d x \geq \int_{1}^{r} \frac{d x}{2^{\alpha}}=\frac{r-1}{2^{\alpha}}$.

Our assertion follows since $\frac{\log ^{\alpha}\left(1+\mathrm{r}^{\mathrm{p}}\right)}{\mathrm{r}-1} \rightarrow 0$ as $\mathrm{r} \rightarrow \infty$.

III. If $(u, v) \in A_{p}$ for some $1 \leq p<\infty$, then

$\int_{\{M f>1\}} M f^{p} u \leq c_{1} \int_{\left\{f>c_{2}\right\}} f^{p} \log \left(1+f^{p}\right) v$.

Proof. Let $\Phi(\mathrm{t})=(\mathrm{t}-1) \chi^{1}(\mathrm{t})$, where $\chi^{1}(\mathrm{t})=\chi_{[1, \infty)}(\mathrm{t})$. Then $\mathrm{a}(\mathrm{t})=\chi^{1}(\mathrm{t})$. We let $\mathrm{b}(\mathrm{s})=\int_{0}^{\mathrm{s}} \frac{\mathrm{a}(\mathrm{t})}{\mathrm{t}} \mathrm{dt}=(\log \mathrm{s}) \chi^{1}(\mathrm{~s})$

Then $\Psi(\mathrm{t})=\int_{0}^{\mathrm{t}} \mathrm{b}(\mathrm{s}) \mathrm{ds} \leq(\mathrm{t} \log \mathrm{t}) \chi^{1}(\mathrm{t})$. By Theorem 1 we get $\int_{\{\mathrm{Mf} \geq 1\}}\left(\mathrm{Mf}^{\mathrm{p}}-1\right) \mathrm{u} \leq \mathrm{c}_{1} \int_{\left\{\mathrm{f} \geq 1 / \mathrm{c}^{\prime}\right\}} \mathrm{f}^{\mathrm{p}} \log \left(\mathrm{c}_{2} \mathrm{f}^{\mathrm{p}}\right) \mathrm{v}$,

where $c^{\prime}=c_{2}^{1 / p}$. By Theorem 2

$\mathrm{u}\{\mathrm{Mf} \geq 1\} \leq \mathrm{c}_{0} \int_{1 / \mathrm{c}_{0}}^{\infty} \mathrm{v}\{\mathrm{f} \geq \mathrm{s}\} \mathrm{s}^{\mathrm{p}-1} \mathrm{ds}=\frac{\mathrm{c}_{0}}{\mathrm{p}} \int_{\mathrm{c}^{\prime \prime}}^{\infty} \mathrm{v}\left\{\mathrm{f}^{\mathrm{p}}>\mathrm{t}\right\} \mathrm{dt} \leq \frac{\mathrm{c}_{0}}{\mathrm{p}} \int_{\left\{\mathrm{f} \geq \mathrm{c}^{\prime \prime}\right\}} \mathrm{f}^{\mathrm{p}} \mathrm{v}$,

where $c^{\prime \prime}=1 /\left(c_{0}^{\mathrm{p}}\right)<1$. Thus we get

$\int_{\{\mathrm{Mf} \geq 1\}} \mathrm{Mf}^{\mathrm{p}} \mathrm{u} \leq \mathrm{c}_{1} \int_{\left\{\mathrm{f} \geq \mathrm{c}_{*}\right\}} \mathrm{f}^{\mathrm{p}}\left(1+\log \left(\mathrm{c}_{2} \mathrm{f}^{\mathrm{p}}\right)\right) \mathrm{v} \leq \mathrm{c}_{1^{\prime}} \int_{\left\{\mathrm{f} \geq \mathrm{c}_{*}\right\}} \mathrm{f}^{\mathrm{p}} \log \left(1+\mathrm{f}^{\mathrm{p}}\right) \mathrm{v}$,

since $1+\log (c x) \leq e c \log (1+x)$ if $e c>1$.

Remark: As a special case, if $(u, v) \in A_{1}$ and $K \subset R^{n}$ is compact, then $\int_{R^{n}} f \log (1+f) v<\infty$ implies $M f \chi_{K} \in L^{1}(u)$. This is a two-weight version of the well-known fact that $M f \chi_{K} \in L^{1}$ , if $\mathrm{f} \in \mathrm{L} \log \mathrm{L}$ [10].

IV. Let $(\mathrm{u}, \mathrm{v}) \in \mathrm{A}_{\mathrm{p}}$ for some $1 \leq \mathrm{p}<\infty$, and let $0<\alpha<1$. Then

$\int_{\{\mathrm{Mf}>1\}}\left(\mathrm{Mf}^{\alpha \mathrm{p}}-1\right) \mathrm{u} \leq \frac{\mathrm{c}_{1}}{1-\alpha} \int_{\left\{\mathrm{c}_{2} \mathrm{f}^{\mathrm{p}}>1\right\}}\left(\mathrm{c}_{2} \mathrm{f}^{\mathrm{p}}-\mathrm{c}_{2}^{\alpha} \mathrm{f}^{\alpha \mathrm{p}}\right) \mathrm{v}$.

Proof. Let $\Phi(\mathrm{t})=\left(\mathrm{t}^{\alpha}-1\right) \chi^{1}(\mathrm{t})$. Then $\mathrm{a}(\mathrm{t})=\alpha \mathrm{t}^{\alpha-1} \chi^{1}(\mathrm{t})$. We set $\mathrm{b}(\mathrm{t})=\alpha \int_{1}^{\mathrm{t}} \mathrm{s}^{\alpha-2} \mathrm{ds} \chi^{1}(\mathrm{t})=\frac{\alpha}{1-\alpha}\left(1-\mathrm{t}^{\alpha-1}\right) \chi^{1}(\mathrm{t})$.

Hence

$\Psi(t)=\frac{\alpha}{1-\alpha} \int_{1}^{t}\left(1-s^{\alpha-1}\right) d s \chi^{1}(t)=\left(\frac{\alpha}{1-\alpha}\left(t-t^{\alpha} / \alpha\right)+1\right) \chi^{1}(t)$.

$>$ From Theorem 1, using $v\left\{c_{2} \mathrm{f}^{\mathrm{p}}>1\right\} \leq \int_{\left\{\mathrm{c}_{2} \mathrm{f}\right.} \mathrm{p}_{>1\}} \mathrm{c}_{2} \mathrm{f}^{\mathrm{p}} \mathrm{v}$, we get the desired inequality.

V. Let $(u, v) \in A_{p}$ for some $1 \leq p<\infty$, and let $0<k<\infty$.

Then $\int_{\{\text {Mf }>1\}}\left(1-\frac{1}{M f^{p}}\right)^{k} u \leq c \int_{\left\{f>1 / c^{\prime}\right\}} f^{p}\left(1-1 /\left(c_{2} f^{p}\right)\right)^{k+1} v, c^{\prime}=c_{2}^{1 / p}$.

Proof. Let $\Phi(\mathrm{t})=(1-1 / \mathrm{t})^{\mathrm{k}} \chi^{1}(\mathrm{t})$.

Then $\mathrm{a}(\mathrm{t})=\mathrm{k}(1-1 / \mathrm{t})^{\mathrm{k}-1} 1 /\left(\mathrm{t}^{2}\right) \chi^{1}(\mathrm{t})$. We set

$b(t)=k \int_{1}^{t}(1-1 / s)^{k-1} \frac{1}{s^{3}} d s \chi^{1}(t) \leq(1-1 / t)^{k} \chi^{1}(t)$.

$>$ From this we see that

$\Psi(\mathrm{t})=\int_{1}^{\mathrm{t}}(1-1 / \mathrm{s})^{\mathrm{k}} \mathrm{ds} \chi^{1}(\mathrm{t}) \leq(1-1 / \mathrm{t})^{\mathrm{k}}(\mathrm{t}-1) \chi^{1}(\mathrm{t})=\mathrm{t}(1-1 / \mathrm{t})^{\mathrm{k}+1} \chi^{1}(\mathrm{t})$, and the inequality follows.

VI. Let $(u, v) \in A_{p}$ for some $1 \leq p<\infty$. Then

$\int_{R^{n}} e^{-1 /\left(M f^{p}\right)} u \leq c_{1} \int_{R^{n}} f^{p} e^{-1 /\left(c_{2} f^{p}\right)} v$

Proof. Let $\Phi(\mathrm{t})=\mathrm{e}^{-1 / \mathrm{t}}, \mathrm{t}>0$ and $\Phi(0)=0$. Then $\mathrm{a}(\mathrm{t})=\mathrm{e}^{-1 / \mathrm{t}} 1 / \mathrm{t}^{2}$ and

$\mathrm{b}(\mathrm{t})=\int_{0}^{\mathrm{t}} \frac{\mathrm{e}^{-1 / \mathrm{s}}}{\mathrm{s}^{3}} \mathrm{ds}=\mathrm{e}^{-1 / \mathrm{t}}\left(\frac{1}{\mathrm{t}}+1\right)$.

$>$ From this

$\Psi(\mathrm{t})=\int_{0}^{\mathrm{t}} \mathrm{e}^{-1 / \mathrm{s}}\left(\frac{1}{\mathrm{~s}}+1\right) \mathrm{ds}=\mathrm{te}^{-1 / \mathrm{t}}$.

Theorem 1 gives the desired integral inequality.

Remark: The factor $\mathrm{f}^{\mathrm{p}}$ in the above inequality cannot be omitted as examples of the type $\mathrm{f}_{\mathrm{N}}=\mathrm{N} \chi_{[0,1]}$ show.

VII. Suppose $a(t)=\Phi^{\prime}(t)$ is convex with $a(0)=0$. If $(\mathrm{u}, \mathrm{v}) \in \mathrm{A}_{\mathrm{p}}$ for some $1 \leq \mathrm{p}<\infty$, then

$\int_{\mathrm{R}^{\mathrm{n}}} \Phi\left(\mathrm{Mf}^{\mathrm{p}}\right) \mathrm{u} \leq \mathrm{c}_{1} \int_{\mathrm{R}^{\mathrm{n}}} \Phi\left(\mathrm{c}_{2} \mathrm{f}^{\mathrm{p}}\right) \mathrm{v}$

Proof. This follows from

$\int_{0}^{\mathrm{t}} \frac{\mathrm{a}(\mathrm{s})}{\mathrm{s}} \mathrm{ds} \leq \int_{0}^{\mathrm{t}} \mathrm{a}^{\prime}(\mathrm{s}) \mathrm{ds}=\mathrm{a}(\mathrm{t})$.

Remark: Examples illustrating (VII) are $\Phi(\mathrm{t})=\mathrm{t}^{2} \mathrm{e}^{\mathrm{t}}, \mathrm{e}^{\mathrm{t}}-\mathrm{t}-1, \sum_{\mathrm{n} \geq 2} \mathrm{a}_{\mathrm{n}} \mathrm{t}^{\mathrm{n}}, \mathrm{a}_{\mathrm{n}} \geq 0$. As an application we will present an inequality involving $\mathrm{e}^{\mathrm{Mf}} \mathrm{p}$.

VIII. If $(u, v) \in A_{p}$ for some $1 \leq p<\infty$, then there exist constants $0<c_{1}, c_{2}<\infty$ such that for every $f: R^{n} \rightarrow R_{+}$

$\int_{\{M f>1\}} e^{M f}{ }^{p} u \leq c_{1} \int_{\left\{c_{2} f^{p}>1\right\}} e^{c_{2} f^{p}} v$

Proof. Let $\Phi(\mathrm{t})=\left(\mathrm{e}^{\mathrm{t}}-\mathrm{te}\right) \chi^{1}(\mathrm{t})$. Then $\mathrm{a}(\mathrm{t})=\left(\mathrm{e}^{\mathrm{t}}-\mathrm{e}\right) \chi^{1}(\mathrm{t})$ and thus from VII 


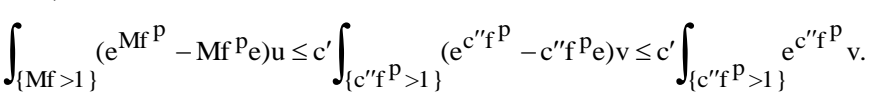

We only need to verify now that

$\int_{\{M f>1\}} M f^{p_{u}} \leq c_{1} \int_{\left\{c_{2} f^{p}>1\right\}} e^{c_{2} f^{p}} v$.

This is easy by letting $\Phi(\mathrm{t})=(\mathrm{t}-1) \chi^{1}(\mathrm{t})$. Then $\mathrm{a}(\mathrm{t})=\chi^{1}(\mathrm{t})$ and thus $\mathrm{b}(\mathrm{t})=\log \mathrm{t} \chi^{1}(\mathrm{t}) \leq \mathrm{e}^{\mathrm{t}} \chi^{1}(\mathrm{t})$.

IX. If $(u, v) \in A_{p}$ for some $1 \leq p<\infty$, then

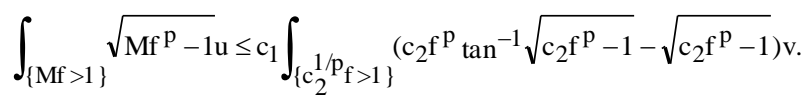

Proof. Let $\chi^{1}(\mathrm{t})=\chi_{[1, \infty)}(\mathrm{t})$, and take $\Phi(\mathrm{t})=\sqrt{\mathrm{t}-1} \chi^{1}(\mathrm{t})$. Then $\mathrm{a}(\mathrm{t})=1 /(2 \sqrt{\mathrm{t}-1}) \chi^{1}(\mathrm{t})$ and

$\mathrm{b}(\mathrm{t})=\int_{1}^{\mathrm{t}} \frac{1}{2 \mathrm{~s}(\mathrm{~s}-1)^{1 / 2}} \mathrm{ds} \chi^{1}(\mathrm{t})=\tan ^{-1} \sqrt{\mathrm{t}-1} \chi^{1}(\mathrm{t})$

Also

$\Psi(\mathrm{t})=\int_{1}^{\mathrm{t}} \tan ^{-1} \sqrt{\mathrm{s}-1} \mathrm{ds} \chi^{1}(\mathrm{t})=\left(\mathrm{t} \tan ^{-1} \sqrt{\mathrm{t}-1}-\sqrt{\mathrm{t}-1}\right) \chi^{1}(\mathrm{t})$.

Theorem 1 gives us the desired integral inequality.

Remark: It is tempting to replace the right side of IX by the more symmetric

$\mathrm{c}_{1} \int_{\left\{\mathrm{c}_{2} \mathrm{f}^{\mathrm{p}}>1\right\}} \sqrt{\mathrm{c}_{2} \mathrm{f}^{\mathrm{p}}-1 \mathrm{v}}$.

Examples of the form $\mathrm{f}_{\mathrm{N}}=\mathrm{N} \chi_{1}$ as $\mathrm{N} \rightarrow \infty$ show that this is not possible.

X. If $(u, v) \in A_{p}$ for some $1 \leq p<\infty$, then

$\int_{\{\text {Mf }>y\}} \log \left(e \frac{\text { Mf }}{y}\right)^{\mathrm{p}} \mathrm{u} \leq \frac{\mathrm{c}_{1}}{\mathrm{y}^{\mathrm{p}}} \int_{\left\{\mathrm{f}>\mathrm{c}_{2} \mathrm{y}\right\}} \mathrm{f}_{\mathrm{v}}$,

with $c_{1}, c_{2}$ independent of $\mathrm{f}$.

Proof. Fix $\mathrm{y}>0$ and let $\mathrm{e}^{\mathrm{p}} \tau=\mathrm{y}^{\mathrm{p}}$. If $\mathrm{a}(\mathrm{t})=(1 / \mathrm{t}) \chi^{\tau}(\mathrm{t})$ and

$\mathrm{b}(\mathrm{t})=\int_{0}^{\mathrm{t}} \frac{\mathrm{a}(\mathrm{s})}{\mathrm{s}} \mathrm{ds}=\int_{\tau}^{\mathrm{t}} \frac{\mathrm{ds}}{\mathrm{s}^{2}} \chi^{\tau}(\mathrm{t}) \leq(1 / \tau) \chi^{\tau}(\mathrm{t})$,

then $\Phi(\mathrm{t})=\log (\mathrm{t} / \tau) \chi^{\tau}(\mathrm{t})$ and $\Psi(\mathrm{t}) \leq(\mathrm{t} / \tau) \chi^{\tau}(\mathrm{t})$. $>$ From Theorem 1 we get

$\int_{\left\{\mathrm{Mf}^{\mathrm{p}}>\tau\right\}} \log \left(\frac{\mathrm{Mf}^{\mathrm{p}}}{\tau}\right) \mathrm{u} \leq \frac{\mathrm{c}^{\prime}}{\tau} \int_{\left\{\mathrm{f}^{\mathrm{p}} \mathrm{c}^{\prime \prime} \tau\right\}} \mathrm{f}_{\mathrm{v}}$

Finally

$\int_{\{\text {Mf }>y\}} \log \left(\mathrm{e}^{\mathrm{p}} \frac{\mathrm{Mf}^{\mathrm{p}}}{\mathrm{y}^{\mathrm{p}}}\right) \mathrm{u} \leq \int_{\{\mathrm{Mf}} \mathrm{p}_{>\tau\}} \log \left(\frac{\mathrm{Mf}^{\mathrm{p}}}{\tau}\right) \mathrm{u} \leq \frac{\mathrm{c}_{1}}{\mathrm{y}^{\mathrm{p}}} \int_{\left\{\mathrm{f}>\mathrm{c}_{2} \mathrm{y}\right\}} \mathrm{f}^{\mathrm{p}} \mathrm{v}$.
Remark: The above inequality is a generalization of the weak-type inequality $\mathrm{u}\{\mathrm{Mf}>\mathrm{y}\} \leq \frac{\mathrm{c}}{\mathrm{y}^{\mathrm{p}}} \int_{\mathrm{R}^{\mathrm{n}}} \mathrm{f}^{\mathrm{p}} \mathrm{v}$.

XI. If $(u, v) \in A_{p}$ and $p<s<r<\infty$, then

$\int_{\{M f>1\}}\left(M f^{r}-M f^{s}\right) u \leq \frac{c_{1}}{\beta-1} \int_{\left\{c_{2} f^{p}>1\right\}}\left(c_{2}^{\alpha} f^{r}-c_{2}^{\beta_{f}}{ }^{s}\right) v$,

where $\alpha=r / p, \beta=s / p$.

Proof. Let $\Phi(\mathrm{t})=\left(\mathrm{t}^{\alpha}-\mathrm{t}^{\beta}\right) \chi^{1}(\mathrm{t})$. Then $\mathrm{a}(\mathrm{t})=\left(\alpha \mathrm{t}^{\alpha-1}-\beta \mathrm{t}^{\beta-1}\right) \chi^{1}(\mathrm{t})$ and

$\mathrm{b}(\mathrm{t})=\left(\frac{\alpha}{\alpha-1} \mathrm{t}^{\alpha-1}-\frac{\beta}{\beta-1} \mathrm{t}^{\beta-1}+\mathrm{c}_{\alpha \beta}\right) \chi^{1}(\mathrm{t})$,

where $c_{\alpha \beta}=\beta /(\beta-1)-\alpha /(\alpha-1)$. Consequently

$\Psi(t)=\left(\frac{t^{\alpha}}{\alpha-1}-\frac{t^{\beta}}{\beta-1}+c_{\alpha \beta} t\right) \chi^{1}(t) \leq\left(\left(\frac{1}{\alpha-1}+c_{\alpha \beta}\right) t^{\alpha}-\frac{1}{\beta-1} t^{\beta}\right)$

$\chi^{1}(t)=\frac{1}{\beta-1}\left(t^{\alpha}-t^{\beta}\right) \chi^{1}(t)$.

Theorem 1 gives the desired inequality.

Remark: If $\mathrm{s}=\mathrm{p}$ above, then using the same type of argument with $\Phi(\mathrm{t})=\left(\mathrm{t}^{\alpha}-\mathrm{t}\right) \chi^{1}(\mathrm{t})$, etc, we get for $(\mathrm{u}, \mathrm{v}) \in \mathrm{A}_{\mathrm{p}}$

$\int_{\{M f>1\}}\left(\mathrm{Mf}^{\mathrm{r}}-\mathrm{Mf}^{\mathrm{p}}\right) \mathrm{u} \leq \frac{\mathrm{c}_{1} \alpha}{\alpha-1} \int_{\left\{\mathrm{c}_{2} \mathrm{f}^{\mathrm{p}}>1\right\}}\left(\mathrm{c}_{2}^{\alpha} \mathrm{f}^{\mathrm{r}}-\mathrm{c}_{2} \mathrm{f}^{\mathrm{p}}\right) \mathrm{v}$.

XII. The fact that $\mathrm{Mf} \notin \mathrm{L}^{1}\left(\mathrm{R}^{\mathrm{n}}\right)$ unless $\mathrm{f}=0$ gives rise to the question for which $\Phi: \mathrm{R}_{+} \rightarrow \mathrm{R}_{+}$is $\Phi(\mathrm{Mf}) \in \mathrm{L}^{1}\left(\mathrm{R}^{\mathrm{n}}\right)$. Let $\mathrm{a}: \mathrm{R}_{+} \rightarrow \mathrm{R}_{+}$be in $\mathrm{L}_{\text {loc }}^{1}((0, \infty))$ and let $\Phi(\mathrm{t})=\int_{0}^{\mathrm{t}} \mathrm{a}(\mathrm{s}) \mathrm{ds}$.

Theorem 10 The following statements are equivalent for $\mathrm{f} \in \mathrm{L}^{\infty} \cap \mathrm{L}^{1}\left(\mathrm{R}^{\mathrm{n}}\right)$ :

$\Phi(\operatorname{Mf}(\mathrm{x})) \in \mathrm{L}^{1}\left(\mathrm{R}^{\mathrm{n}}\right)$,

$\int_{0}^{\mathrm{s}} \frac{\mathrm{a}(\mathrm{t})}{\mathrm{t}} \mathrm{dt}<\infty, 0<\mathrm{s}<\infty$.

Proof. (2) $\rightarrow$ (1). Since $|\{\mathrm{Mf}>\mathrm{t}\}| \leq \mathrm{c}_{0} / \mathrm{t}\|\mathrm{f}\|_{1}$ and $\mathrm{Mf}$ is $(\infty, \infty)$, we get

$\int_{\mathrm{R}^{\mathrm{n}}} \Phi(\operatorname{Mf}(\mathrm{x})) \mathrm{dx}=\int_{0}^{\|\mathrm{f}\|_{\infty}}|\{\mathrm{Mf}>\mathrm{t}\}| \mathrm{a}(\mathrm{t}) \mathrm{dt} \leq \mathrm{c}\|\mathrm{f}\|_{1} \int_{0}^{\|\mathrm{f}\|_{\infty}} \frac{\mathrm{a}(\mathrm{t})}{\mathrm{t}} \mathrm{dt}$.

$(1) \rightarrow(2)$. We may assume that $a(t) \neq 0$ on any interval $(0, \varepsilon))$ and $\mathrm{f} \neq 0$. By Lemma 3, $\frac{1}{\mathrm{t}} \int_{\{\mathrm{f}>\mathrm{t}\}} \mathrm{f}(\mathrm{x}) \mathrm{dx} \leq \mathrm{c}|\{\mathrm{Mf}>\mathrm{t}\}|$, and thus for $\mathrm{f} \in \mathrm{L}^{\infty} \cap \mathrm{L}^{1}\left(\mathrm{R}^{\mathrm{n}}\right)$

$\infty>\int_{\mathrm{R}^{\mathrm{n}}} \Phi(\operatorname{Mf}(\mathrm{x})) \mathrm{dx}=\int_{0}^{\|\mathrm{f}\|_{\infty}}|\{\mathrm{Mf}>\mathrm{t}\}| \mathrm{a}(\mathrm{t}) \mathrm{dt} \geq \mathrm{c} \int_{0}^{\|\mathrm{f}\|_{\infty}} \frac{\mathrm{a}(\mathrm{t})}{\mathrm{t}}$

$\int_{\{f>t\}} f(x) d x d t=c \int_{R^{n}} \int_{0}^{f(x)} \frac{a(t)}{t} f(x) d t d x=c \int_{R^{n}} \Psi(f(x)) f(x) d x$, 
where $\Psi(r)=\int_{0}^{r} \frac{a(t)}{t} d t$. Therefore, $\Psi(f(x)) f(x)<\infty$, a.e $x$, and hence $\Psi(f(x))<\infty$, a.e. $x$.

Incidentally, we have established the following inequality:

$c_{1} \int_{R^{n}} \Psi(f(x)) f(x) d x \leq \int_{R^{n}} \Phi(\operatorname{Mf}(x)) d x \leq c_{2} \Psi\left(\|f\|_{\infty}\right)\|f\|_{1}$.

XIII. Let $\mathrm{a}: \mathrm{R}_{+} \rightarrow \mathrm{R}_{+}, \mathrm{b}(\mathrm{s})=\int_{0}^{\mathrm{s}} \frac{\mathrm{a}(\mathrm{t})}{\mathrm{t}} \mathrm{dt}$ and $\Phi(\mathrm{t})=\int_{0}^{\mathrm{t}} \mathrm{a}(\mathrm{s}) \mathrm{ds}$. If $1 \leq \mathrm{p}, \mathrm{q}<\infty$ and $(\mathrm{u}, \mathrm{v}) \in \mathrm{A}_{\mathrm{p}}$, then

$\int_{R^{n}} \Phi\left(M f^{p}\right) u \leq c_{1} \int_{R^{n}} \Psi_{p, q}\left(c_{2} f^{q}\right) v$

where $\Psi_{\mathrm{p}, \mathrm{q}}(\mathrm{t})=\int_{0}^{\mathrm{t}^{\mathrm{p} / \mathrm{q}}} \mathrm{b}(\mathrm{s}) \mathrm{ds}$.

Proof. This follows from Theorem 1 since $\Psi_{\mathrm{p}, \mathrm{q}}(\mathrm{t})=\Psi\left(\mathrm{t}^{\mathrm{p} / \mathrm{q}}\right)$.

Remark: Theorem 1 deals with functions $\Phi, \Psi$ nondecreasing. It is sometimes convenient to have a version of Theorem 1 with $\Phi, \Psi$ non-increasing.

Let $\mathrm{a}: \mathrm{R}_{+} \rightarrow \mathrm{R}_{+}$and let $\Phi(\mathrm{t})=\int_{\mathrm{t}}^{\infty} \mathrm{a}(\mathrm{s}) \mathrm{ds}$. The function $\mathrm{b}: \mathrm{R}_{+} \rightarrow \mathrm{R}_{+}$is related to a by

$\int_{\mathrm{s}}^{\infty} \operatorname{ta}(\mathrm{t}) \mathrm{dt} \leq \mathrm{c}^{\prime} \mathrm{b}\left(\mathrm{c}^{\prime \prime} \mathrm{s}\right), 0<\mathrm{s}<\infty$

Finally, let

$\Psi(t)=\int_{t}^{\infty} \frac{b(s)}{s^{2}} d s$.

Theorem 11 The following statements are equivalent for $1 \leq \mathrm{p}<\infty$.

(6) Whenever $\Phi$ and $\Psi$ are related as above, then for every $f: R^{n} \rightarrow R_{+}$

$\int_{R^{n}} \Phi\left(\frac{1}{M^{p}}\right) \mathrm{u} \leq \mathrm{c}_{1} \int_{\mathrm{R}^{\mathrm{n}}} \Psi\left(\frac{\mathrm{c}_{2}}{\mathrm{f}^{\mathrm{p}}}\right) \mathrm{v}$,

where the constants $c_{1}, c_{2}$ depend only on $\mathrm{c}^{\prime}, \mathrm{c}^{\prime \prime}$ and $\mathrm{p}$.

(7) $(u, v) \in A_{p}$.

Proof. The change of variables $\mathrm{s} \rightarrow 1 / \mathrm{s}$ shows that condition (2) of Theorem 1 is equivalent with condition (6): $\Phi(\mathrm{t}), \Psi(\mathrm{t})$ satisfy (6) if and only if $\Phi_{*}(\mathrm{t})=\Phi(1 / \mathrm{t}), \Psi_{*}(\mathrm{t})=\Psi(1 / \mathrm{t})$ satisfy (2) of Theorem 1.
As an example let $\Phi(t)=\int_{t}^{\infty} e^{-s} d s$. An easy calculation shows that we get VI. Another interesting example is $\Phi(t)=\left(1-t^{\alpha}\right) \chi_{1}(t), \quad 0<\alpha<\infty, \quad$ where $\quad \chi_{1}(t)=\chi_{[0,1]}(t)$. Then $\mathrm{a}(\mathrm{t})=\alpha \mathrm{t}^{\alpha-1} \chi_{1}(\mathrm{t})$ and $\mathrm{s}$

$b(t)=\frac{\alpha}{\alpha+1}\left(1-t^{\alpha+1}\right) \chi_{1}(t)$.

Thus

$$
\begin{aligned}
& \Psi(\mathrm{t})=\int_{\mathrm{t}}^{\infty} \frac{\mathrm{b}(\mathrm{s})}{\mathrm{s}^{2}} \mathrm{ds} \chi_{1}(\mathrm{t})=\left\{\frac{\alpha}{\alpha+1}\left(1 / \mathrm{t}+\mathrm{t}^{\alpha} / \alpha\right)-1\right\} \chi_{1}(\mathrm{t}) . \\
& \text { If }(\mathrm{u}, \mathrm{v}) \in \mathrm{A}_{\mathrm{p}} \text { for some } 1 \leq \mathrm{p}<\infty \text {, Theorem } 7 \text { gives } \\
& \int_{\{\mathrm{Mf}>1\}}\left(1-\frac{1}{\mathrm{Mf}}\right) \mathrm{u} \leq \mathrm{c}_{1} \int_{\left\{\mathrm{c}_{2}^{1 / \mathrm{p}}<\mathrm{f}\right\}}\left(\frac{\mathrm{f}^{\mathrm{p}}}{\mathrm{c}_{2}}+\frac{\mathrm{c}_{2}^{\alpha}}{\alpha \mathrm{f}^{\alpha \mathrm{p}}}\right) \mathrm{v}-\mathrm{c}_{1} \mathrm{v}\left\{\mathrm{f}>\mathrm{c}_{2}^{1 / \mathrm{p}}\right\} .
\end{aligned}
$$

\section{CONFLICT OF INTEREST}

The authors confirm that this article content has no conflicts of interest.

\section{ACKNOWLEDGEMENT}

None declared.

\section{REFERENCES}

[1] Cruz-Uribe D, Martell J, Pérez C. Weights, Extrapolation, and the Theory of Rubio de Francia. In: Ball JA, Dym H, Kaashock MA, Langes H, Tretter C, Eds. Operator Theory. Advances and Applications. Basal: Birkhäuser 2010; p. 215.

[2] Cruz-Uribe D, Neugebauer CJ. The structure of reverse Hölder classes. Trans Am Math Assoc 1995; 347: 2941-59.

[3] Garcia-Cuerva J, Rubio De Francia JL. Weighted norm inequalities and related topics, North-Holland Mathematics Studies. Netherlands: Elsevier 1985; p. 116.

[4] Muckenhoupt B. Weighted norm inequalities for the Hardy maximal function. Trans Am Math Assoc 1972; 165: 207-26.

[5] Muckenhoupt B, Wheeden R. Two weight function norm inequalities for the Hardy-Littlewood maximal function and the Hilbert transform. Stud Math 1976; 60: 279-94.

[6] Neugebauer CJ. Orlicz-type integral inequalities for operators. J Korean Math 2001; 38: 163-76.

[7] Neugebauer CJ. Weighted norm inequalities for averaging operators of monotone functions. Publicacions Matema $t^{\prime}$ iques 1991; 35: 429-47.

[8] Neugebauer CJ, Leckband MA. A general maximal operator and the $A_{p}$ condition. Trans Am Math Assoc 1983; 275: 821-31.

[9] Neugebauer CJ, Leckband MA. Weighted iterates and variants of the Hardy-Littlewood maximal operator. Trans Am Math Assoc 1983; 275(1): 51-61.

[10] Stein EM. Singular integrals and differentiability properties of functions. Princeton, NJ: Princeton University Press 1970.

[11] Torchinsky A. Real-variable methods in harmonic analysis. USA: Academic Press 1986. 